Article

\title{
Alternative Access to Functionalized 2,8-Ethanonoradamantane Derivatives
}

\author{
Pelayo Camps ${ }^{1, *}$, Tània Gómez ${ }^{1}$, Ane Otermin ${ }^{1}$ and Mercè Font-Bardia ${ }^{2,3}$ \\ 1 Laboratori de Química Farmacèutica (Unitat Associada al CSIC), Facultat de Farmàcia i Ciències de la \\ Alimentació and Institut de Biomedicina (IBUB), Universitat de Barcelona, Av. Joan XXIII 27-31, \\ Barcelona 08028, Spain; tania_gomeznadal@hotmail.com (T.G.); aneotermin@gmail.com (A.O.) \\ 2 Departament de Mineralogia, Petrologia i Geologia Aplicada, Universitat de Barcelona, Martí Franquès s/n, \\ Barcelona 08028, Spain; mercef@ccit.ub.edu \\ 3 Unitat de Difracció de RX, Centres Científics i Tecnològics de la Universitat de Barcelona (CCiTUB), \\ Solé i Sabarís 1-3, Barcelona 08028, Spain \\ * Correspondence: camps@ub.edu; Tel.: +34-934-024-536
}

Academic Editor: Roman Dembinski

Received: 13 May 2017; Accepted: 25 May 2017; Published: 31 May 2017

\begin{abstract}
Methoxycarbonyl)-N-methyl-1,3a,5,6,7,7a-hexahydro-4H-1,4,6-(epiethane[1,1,2]triyl) indene-4,9-dicarboximide has been prepared through a modification of a previous synthetic sequence, in which the benzyloxymethyl hydroxyl protecting group has been replaced by methoxymethyl, to avoid the apparent formation of a benzyl ester derivative as a side product. The overall yield of the new synthetic sequence is comparable to the previous one. Two advantages of the new procedure are: (a) no benzyl ester was formed and (b) a stereoisomeric mixture of syn-and anti-alcohols at the beginning of the synthetic sequence could be separated and the rest of the synthesis could be carried out with the main syn-stereoisomer instead of the corresponding stereoisomeric mixture as it was the case in the previous process. Additionally, several functional 2,8-ethanonoradamantane derivatives have been prepared.
\end{abstract}

Keywords: polycycles; intramolecular cycloaddition; retrocycloaddition; domino reactions; X-ray diffraction

\section{Introduction}

Some time ago, the synthesis of the functionalized polycycle $\mathbf{1 3}$ as a new scaffold for the preparation of compounds with potential biological activity was described [1]. Later on, improvements of the synthesis of $\mathbf{1 3}$ were performed [2,3], the highly-optimized synthetic route to this compound is shown in Scheme 1. During purification of this compound, implying a sublimation process, the presence of a benzyl ester derivative, probably 14, in the non-sublimed residue was suggested by ${ }^{1} \mathrm{H}-\mathrm{NMR}$. To solve this problem, an alternative synthesis of polycycle $\mathbf{1 3}$ was planned in which the methoxymethyl hydroxyl-protecting group would be used instead of the benzyloxymethyl one (Scheme 2). 


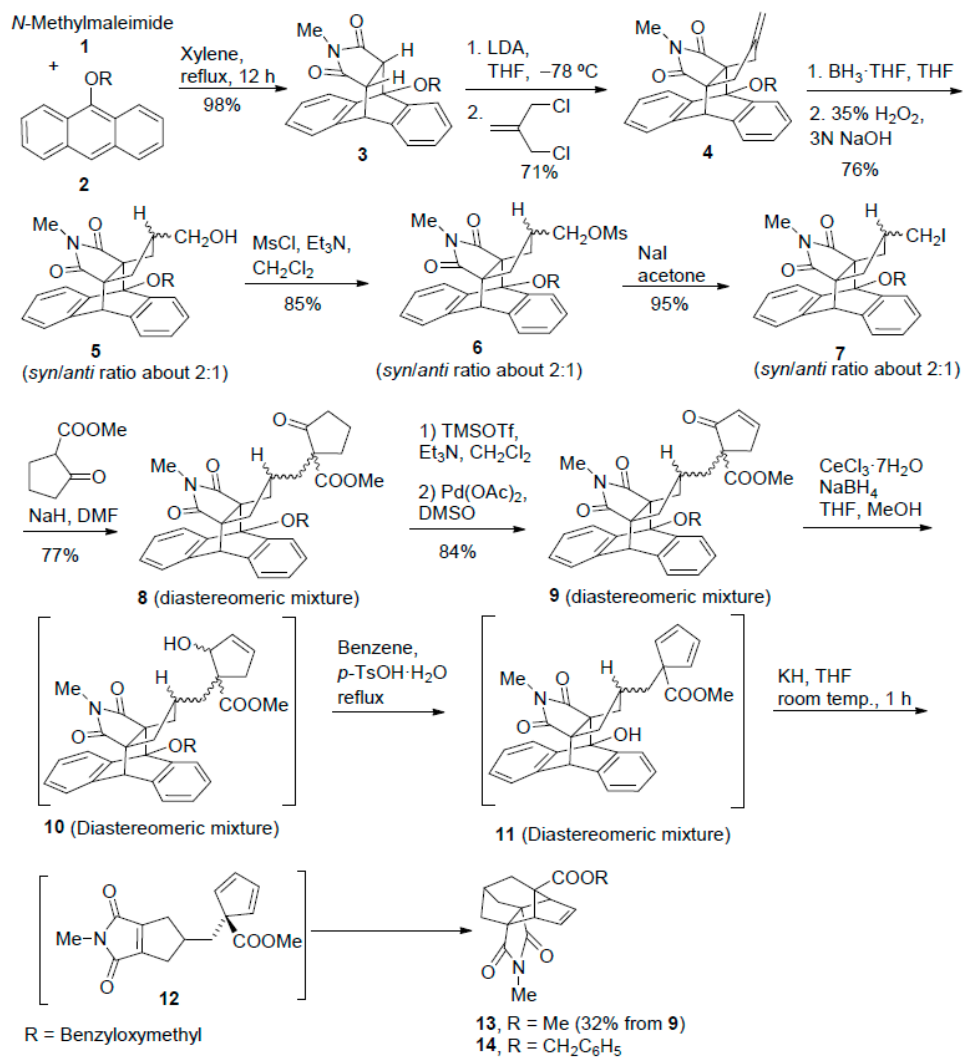

Scheme 1. Previously improved synthesis of compound 13.
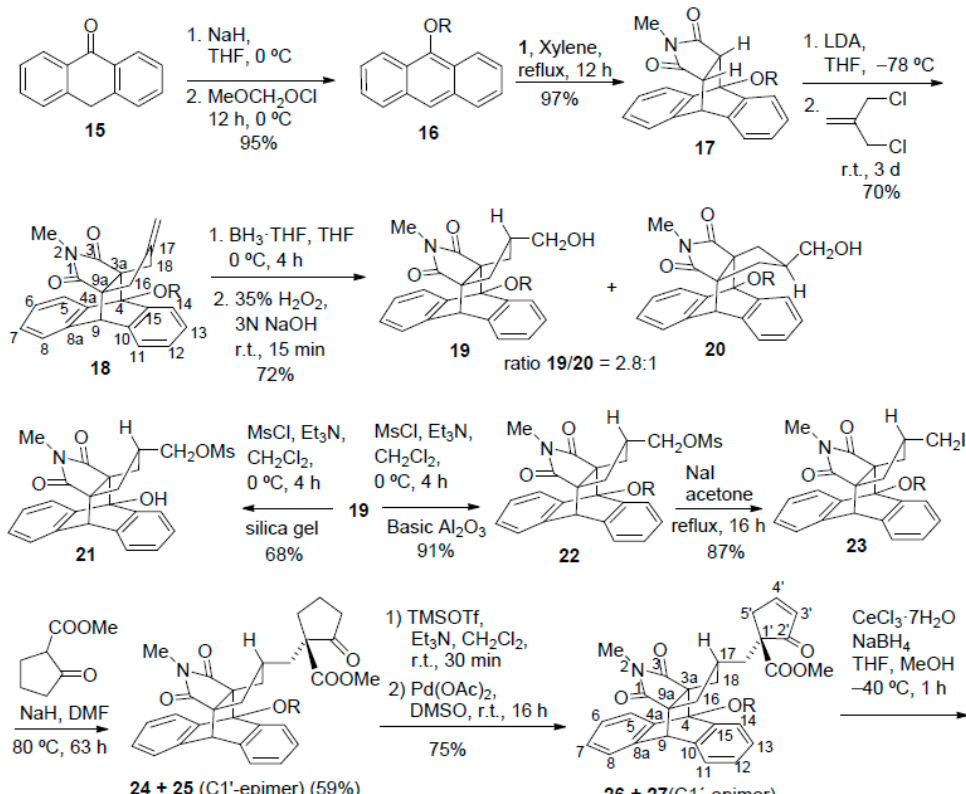

$24+25$ (C1'-epimer) (59\%)

$26+27$ (C1'-epimer)

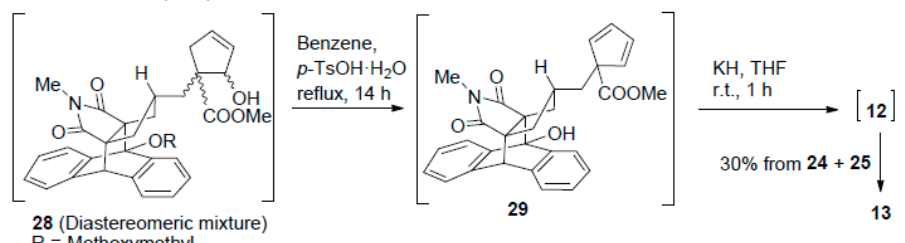

28 (Diastereomeric mixture

Scheme 2. Alternative synthesis of tetracycle 13, using methoxymethyl as a hydroxyl-protecting group. 


\section{Results and Discussion}

The reaction of anthrone with methoxymethyl chloride, using $\mathrm{NaH}$ as the base, and following a procedure similar to that used to prepare anthracene 2 [4,5], gave 9-methoxymethylanthracene 16. The reaction of $N$-methylmaleimide 1 with anthracene 16 gave the corresponding Diels-Alder adduct 17. The reaction of $\mathbf{1 7}$ with 3-chloro-2-chloromethyl-1-propene using lithium diisopropylamide as the base gave the methylenecyclopentane derivative 18 in 70\% yield. Hydroboration of 18 with the borane-THF complex in THF followed by hydrogen peroxide oxidation of the intermediate boranes under strongly basic conditions gave a stereoisomeric mixture of alcohols 19 and 20 in a ratio 19/20 = 2.8:1 $\left({ }^{1} \mathrm{H}-\mathrm{NMR}\right)$. Worthy of note, this mixture was separated by silica gel column chromatography and each stereoisomer could be fully characterized. In the previous synthetic sequence using the benzyloxymethyl hydroxyl protecting group, the stereoisomeric mixture of alcohols 5 could not be separated and, consequently, the next steps in that synthetic sequence were carried out with the corresponding stereoisomeric mixtures. In the present work, however, the synthetic sequence of Scheme 2 was carried out with the main stereoisomeric racemate syn-alcohol 19.

As previously observed for the main syn-stereoisomer of 5 [2], the ${ }^{1} \mathrm{H}-\mathrm{NMR}$ data of the syn-alcohol 19 suggests that exists mainly in the shown envelope conformation, the $16-\mathrm{H}_{n}(\delta=0.92 \mathrm{ppm})$ and $18-\mathrm{H}_{\mathrm{n}}(\delta=1.14 \mathrm{ppm})$ protons appearing quite shielded by the aromatic ring as triplets $\left({ }^{2} J_{(\mathrm{HH})}={ }^{3} J_{(\mathrm{HH})}\right.$ $=12.8 \mathrm{~Hz}$ ) due to the similar value of the geminal and vicinal (dihedral angle close to $180^{\circ}$ ) coupling constants. The $16-\mathrm{H}_{\mathrm{x}}(\delta=2.16 \mathrm{ppm})$ and $18-\mathrm{H}_{\mathrm{x}}(\delta=2.28 \mathrm{ppm})$ protons of alcohol 19 are not so affected by the aromatic ring. In the case of the minor anti-stereoisomer $20,17-\mathrm{H}$ is the more shielded proton $(\delta=1.09-1.20 \mathrm{ppm})$. In this case, the $16-\mathrm{H}_{\mathrm{n}}(\delta=1.71 \mathrm{ppm})$ and $18-\mathrm{H}_{\mathrm{n}}(\delta=2.12 \mathrm{ppm})$ protons are not significantly affected by the aromatic ring. However, the vicinal coupling constants of $16-\mathrm{H}_{\mathrm{x}}$ $\left(\delta=1.83-1.89 \mathrm{ppm},{ }^{3} J_{(\mathrm{H}, \mathrm{H})}=6.8 \mathrm{~Hz}\right)$ and $18-\mathrm{H}_{\mathrm{x}}\left(\delta=1.79-1.85 \mathrm{ppm},{ }^{3} J_{(\mathrm{H}, \mathrm{H})}=6.8 \mathrm{~Hz}\right)$, suggest the contribution of other conformations apart from that shown in Scheme 3, for which a higher value of the above vicinal coupling constants would be expected.
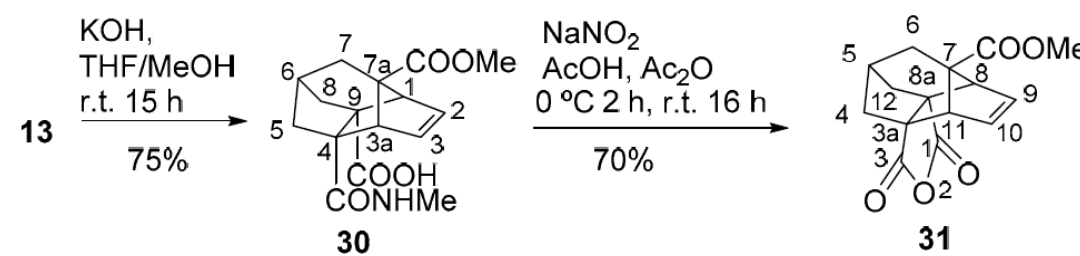

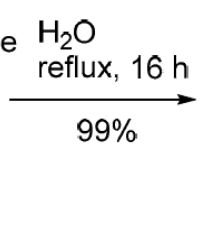

31

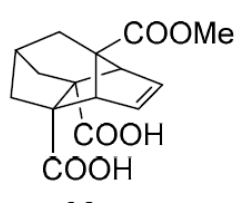

32

Scheme 3. Several functional group transformations from imide ester 13.

The mesylation of alcohol 19 under the usual conditions gave, after purification of the crude product by silica gel column chromatography, mesylate 21, in which the methoxymethyl-protecting group had been hydrolyzed. However, purification of the crude mesylate by basic aluminum oxide column chromatography gave the desired mesylate 22 in high yield. The reaction of mesylate 22 with powdered sodium iodide in refluxing acetone gave iodide $\mathbf{2 3}$ in good yield. In one occasion, the methoxymethyl group of compound 23 was hydrolyzed on standing $\left({ }^{1} \mathrm{H}-\mathrm{NMR}\right)$, thus, it is recommended not to stock this compound. In the sequence of Scheme 1, in which the benzyloxymethyl was the hydroxyl-protecting group, no hydrolysis at the level of the corresponding mixtures of mesylates 6 or iodides 7 was ever observed. Reaction of iodide 23 with the sodium salt of methyl 2-oxocyclopentanecarboxylate in DMF gave an essentially 1:1 steroisomeric mixture of the substitution product 24 and the $\mathrm{C}^{\prime}$ epimer 25 in 59\% yield, after column chromatography. Some elimination product 18 was also isolated in 17\% yield. After repeated crystallization of this mixture from EtOAc/hexane, stereoisomer $\mathbf{2 4}$ was obtained in pure form. The structure of $\mathbf{2 4}$ was established by X-ray diffraction analysis (Figure 1). Although compounds $\mathbf{1 7}$ to $\mathbf{2 7}$ of this synthetic sequence are racemic, the unit cell of the crystal used for the X-ray analysis of $\mathbf{2 4}$ contained four molecules of the same enantiomer whose absolute configuration could not be established from the X-ray data [6]. 
Reaction of the stereoisomeric mixture of keto esters $\mathbf{2 4}$ and $\mathbf{2 5}$ with trimethylsilyl triflate gave the corresponding mixture of trimethylsilyl enol ethers that was oxidized without purification with $\mathrm{Pd}(\mathrm{OAc})_{2}$ in DMSO [7] to give the stereoisomeric mixture of cyclopentenones $\mathbf{2 6}$ and $\mathbf{2 7}$, in 75\% overall yield. For characterization purposes, the same transformation was carried out with pure stereoisomer 24, thus, obtaining pure stereoisomer 26.

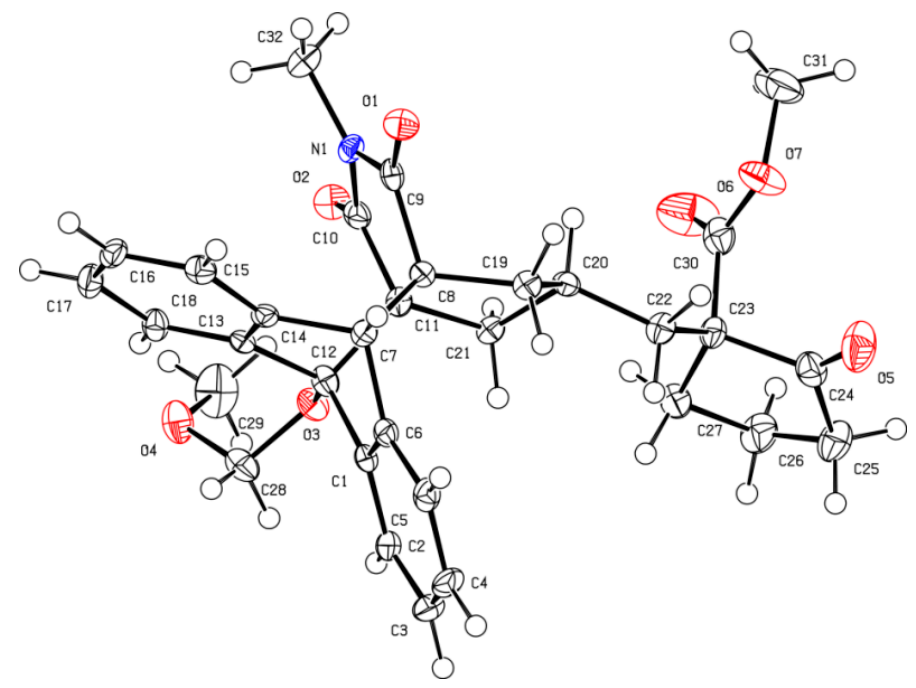

Figure 1. ORTEP representation of compound 24.

Transformation of the stereoisomeric mixture of $\mathbf{2 6}$ and $\mathbf{2 7}$ to polycycle $\mathbf{1 3}$ was carried out without isolating any of the intermediates, as was the case in the sequence of Scheme 1 . Thus, reduction of the 26 and 27 mixture with $\mathrm{NaBH}_{4}$ in the presence of $\mathrm{CeCl}_{3} \cdot 7 \mathrm{H}_{2} \mathrm{O}$ (Luche conditions) [8] gave the diastereomeric mixture of allylic alcohols $\mathbf{2 8}$ which on heating in refluxing benzene in the presence of a catalytic amount of $p$-toluenesulfonic acid was dehydrated with simultaneous deprotection of the methoxymethyl group to compound 29. The oxide anion accelerated retro Diels-Alder reaction of 29 leading to anthrone and maleimide 12 was carried out as usual by treatment with KH in THF [9]. Maleimide 12 experience an intramolecular Diels-Alder reaction producing imide $\mathbf{1 3}$ in $30 \%$ overall yield from the mixture of $\mathbf{2 4}$ and $\mathbf{2 5}$. The overall yield of the synthetic sequence of Scheme $2(5.1 \%)$ is comparable to that of Scheme 1 (7.2\%). As expected, no formation of 14 was observed in this case and, worthy of note, the sequence of Scheme 2 made possible the characterization of all of the intermediates as pure racemates. The lower stability of the methoxymethyl hydroxyl-protecting group compared with the benzyloxymethyl required using neutral aluminum oxide instead of silica gel during the column chromatography purification.

Additionally, the imide function of compound $\mathbf{1 3}$ has been transformed into the corresponding diacid (compound 32, Scheme 3), a transformation that is worth of mention. Thus, basic hydrolysis of 13 took place smoothly at room temperature leading cleanly to the amide acid $\mathbf{3 0}$. This result was surprising taking into account that both $\alpha$-carbon atoms of the imide function are quaternary. In fact, only a closely-related example of this kind of hydrolysis has been previously described, although in this case the reaction was carried out at $50{ }^{\circ} \mathrm{C}$ [10]. Under these conditions the ester function, whose $\alpha$-carbon atom is also quaternary, was not hydrolyzed. We consider that the easy hydrolysis of imide 13 might take place as follow: (i) intramolecular retro-Diels-Alder to revert to maleimide 12; (ii) fast basic hydrolysis of maleimide 12, in accord with our previous experience with related maleimides [2]; and (iii) intramolecular Diels-Alder reaction to give 30. Under more forcing basic conditions the ester function of $\mathbf{3 0}$ was fully hydrolyzed while only partial hydrolysis of the amide function was observed. However, amide acid 30 was transformed into anhydride 31 by the reaction with $\mathrm{NaNO}_{2}$ in a 1:1 mixture of $\mathrm{AcOH}$ and $\mathrm{Ac}_{2} \mathrm{O}$ at room temperature. It is known that $\mathrm{N}$-alkyl-N-nitrosoamides decompose 
thermally to alkyl esters [11,12]. In the present case, the expected ester from the decomposition of the $N$-methyl-N-nitrosoamide, on reaction with the neighbor carboxylate under the reaction conditions would give anhydride 31. Alternatively, addition of the carboxylate group to the carbonyl of the neighbor $N$-nitrosoamide followed by elimination of the $N$-methyl- $N$-nitrosoamide anion would give anhydride 31. Following a related procedure [13], heating anhydride 31 in water under reflux, diacid 32 was obtained in high yield.

\section{Materials and Methods}

\section{General}

Melting points were determined in open capillary tubes with a MFB 595010M Gallenkamp melting point apparatus (Weiss Gallenkamp, Loughborough, UK). All new compounds were fully characterized by their analytical [melting point, elemental analysis and/or accurate mass measurement, spectroscopic data (IR, ${ }^{1} \mathrm{H}-\mathrm{NMR}$ and ${ }^{13} \mathrm{C}-\mathrm{NMR}$, see supplementary)] and, in the case of compound 24, also X-ray diffraction analysis. Assignments given for the NMR spectra are based on DEPT, COSY, ${ }^{1} \mathrm{H} /{ }^{13} \mathrm{C}$ single quantum correlation (gHSQC sequence) and ${ }^{1} \mathrm{H} /{ }^{13} \mathrm{C}$ multiple bond correlation (gHMBC sequence) spectra and by comparison with previous assignments for the benzyloxymethyl series. ${ }^{1} \mathrm{H}-\mathrm{NMR}$ and ${ }^{13} \mathrm{C}-\mathrm{NMR}$ spectra were recorded on a Varian Mercury $400\left(400 \mathrm{MHz}\right.$ for ${ }^{1} \mathrm{H}$ and $100.6 \mathrm{MHz}$ for ${ }^{13} \mathrm{C}$, Varian, Palo Alto, CA, USA) spectrometer. Unless otherwise stated, the NMR spectra have been performed in $\mathrm{CDCl}_{3}$. Chemical shifts $(\delta)$ are reported in parts per million related to internal TMS or $\mathrm{CDCl}_{3}$ for ${ }^{1} \mathrm{H}$ - and ${ }^{13} \mathrm{C}-\mathrm{NMR}$, respectively. Multiplicities are reported using the following abbreviations: $\mathrm{s}$, singlet; $\mathrm{d}$, doublet; $\mathrm{t}$, triplet; $\mathrm{m}$, multiplet; br, broad; or their combinations. IR spectra were registered on a FTIR Perkin-Elmer Spectrum RX1 spectrometer (Perkin-Elmer, Seer Green, UK) using the attenuated total reflectance (ATR) technique. Absorption values are given as wavenumbers $\left(\mathrm{cm}^{-1}\right)$, the intensity of the absorptions are given as strong (s), medium (m) or weak (w). IR and NMR spectra of all new compounds are provided in the Supplementary Materials section of this paper.High resolution mass spectra (HRMS) were carried out at the Mass Spectrometry Unity of the Centres Científics i Tecnològics of the Universitat de Barcelona (CCiTUB, Barcelona, Spain) and are reported as $\mathrm{m} / \mathrm{z}$. LC/MSD-TOF spectrometer with electrospray ionization (ESI-TOF-MS) from Agilent Technologies (Santa Clara, CA, USA) was used. The elemental analyses were carried out at the IQAC (CSIC) of Barcelona, Spain, in elemental microanalyzers (A5) model Flash 1112 series from Thermofinnigan for $(\mathrm{C}, \mathrm{H}, \mathrm{N})$ determinations (Thermo Fisher Scientific SL, Barcelona, Spain) and in a titroprocessor Methrom model 808 (Massó Analítica, Barcelona, Spain) for the halogen determination. For the flash column chromatography, silica gel $60 \mathrm{AC}\left(35-70 \mu \mathrm{m}\right.$, SDS, reference 2000027) or neutral $\mathrm{Al}_{2} \mathrm{O}_{3}$ (50-200 $\mu \mathrm{m})$ was used. The eluents employed are reported as volume/volume percentages. Thin-layer chromatography (TLC) was performed on aluminum-backed sheets with silica gel 60 F254 (Sigma Aldrich Química, S.L., Madrid, Spain, Merck reference 1.05554) and spots were visualized with UV light or a $1 \%$ aqueous solution of $\mathrm{KMnO}_{4}$. X-ray diffraction analysis of compounds 24 was performed in a Bruker D8 Venture diffractometer at the CCiTUB of the University of Barcelona (Bruker Española S.A., Madrid, Spain). The compounds and reagents were purchased to the following companies: methyl 2-oxocyclopentanecarboxylate, methyl chloromethyl ether, silica gel, 60\% $\mathrm{NaH}$ and 30\% $\mathrm{KH}$, both in mineral oil, and $p$-toluenesulfonic acid to Sigma-Aldrich; anthrone and $\mathrm{Pd}(\mathrm{OAc})_{2}$ to Alfa Aesar; $\mathrm{N}$-methylmaleimide and $\mathrm{NaBH}_{4}$ to TCI; 3-chloro-2-chloromethyl-1-propene to Secant Chemicals, Inc., Winchendon, MA, USA; borane THF complex, $n$-BuLi in hexanes, neutral aluminum oxide Brockman I (50-200 $\mu \mathrm{m})$, methanesulfonyl chloride, and NaI to ACROS Organics; diisopropylamine and 35\% $\mathrm{H}_{2} \mathrm{O}_{2}$ to Panreac; trimethylsilyl triflate, $\mathrm{CeCl}_{3} \cdot 7 \mathrm{H}_{2} \mathrm{O}$, and $\mathrm{NaNO}_{2}$ to Fluka. All of them were used without further purification.

9-(Methoxymethoxy)anthracene (16). $\mathrm{NaH}$ (60\% in mineral oil, $3.09 \mathrm{~g}, 77 \mathrm{mmol}$ ) was added in portions to a cold $\left(0{ }^{\circ} \mathrm{C}\right.$, ice-water bath) solution of anthrone $15(10.0 \mathrm{~g}, 51.5 \mathrm{mmol})$ in anhydrous $\mathrm{THF}(600 \mathrm{~mL})$ 
and the mixture was stirred for $45 \mathrm{~min}$ at this temperature. Methoxymethyl chloride $(5.87 \mathrm{~mL}, 6.22 \mathrm{~g}$, $77.3 \mathrm{mmol}$ ) were added at $0{ }^{\circ} \mathrm{C}$ and the mixture was stirred for $12 \mathrm{~h}$ at room temperature. Water $(250 \mathrm{~mL})$ and EtOAc $(300 \mathrm{~mL})$ were added, the organic phase was separated and the aqueous one was extracted with EtOAc $(2 \times 300 \mathrm{~mL})$. The combined organic phases were washed with water $(250 \mathrm{~mL})$ and brine $(250 \mathrm{~mL})$, dried (anhydrous $\left.\mathrm{Na}_{2} \mathrm{SO}_{4}\right)$ and concentrated in vacuo to give $16(11.6 \mathrm{~g}, 95 \%$ yield). An analytical sample of $\mathbf{1 6}(105 \mathrm{mg})$ was obtained as yellow solid by crystallization of a sample of the above product $(300 \mathrm{mg})$ from a mixture of hexane $(5 \mathrm{~mL})$ and EtOAc $(2 \mathrm{~mL})$. m.p. $78-79{ }^{\circ} \mathrm{C}$ (EtOAc/hexane); ${ }^{1} \mathrm{H}-\mathrm{NMR}\left(400 \mathrm{MHz}_{\mathrm{CDCl}}\right): \delta=3.76\left(\mathrm{~s}, 3 \mathrm{H}, \mathrm{OCH}_{3}\right), 5.38\left(\mathrm{~s}, 2 \mathrm{H}, \mathrm{OCH}_{2} \mathrm{O}\right), 7.45-7.52$ [complex signal, 4H, 2(7)-H and 3(6)-H], 7.98-8.01 [m, 2H, 4(5)-H], 8.26 (s, 1H, 10-H), 8.31-8.34 ppm $[\mathrm{m}, 2 \mathrm{H}, 1(8)-\mathrm{H}] ;{ }^{13} \mathrm{C}-\mathrm{NMR}\left(100.6 \mathrm{MHz}, \mathrm{CDCl}_{3}\right): \delta=58.1\left(\mathrm{CH}_{3}, \mathrm{OCH}_{3}\right), 101.2\left(\mathrm{CH}_{2}, \mathrm{OCH}_{2} \mathrm{O}\right), 122.6$ [CH, C1(8)], 122.7 (CH, C10), 125.0 [C, C8a(9a)], 125.3 [CH, C2(7)], 125.5 [CH, C3(6)], 128.3 [CH, C4(5)], 132.3 (C, C4a(4b)], 149.7 ppm (C, C9); IR (ATR): ̌̌ = 1675 (w), 1343 (m), 1309 (m), 1285 (m), 1157 (m), 1098 (m), 1040 (s), 1008 (m), 933 (s), 924 (s), $884(\mathrm{~m}), 846$ (m), 791 (m), 732 (s), 693 (m), $658 \mathrm{~cm}^{-1}(\mathrm{~m})$; accurate mass measurement: $m / z$ calcd. for $\mathrm{C}_{16} \mathrm{H}_{14} \mathrm{O}_{2}+\mathrm{H}^{+}$: 239.1067; found: 239.1056; elemental analysis calcd. (\%) for $\mathrm{C}_{16} \mathrm{H}_{14} \mathrm{O}_{2}$ : C 80.65, $\mathrm{H}$ 5.92; found: C 80.65, $\mathrm{H} 5.84$.

4-(Methoxymethoxy)-2-methyl-3a,4,9,9a-tetrahydro-4,9[1',2']benzeno-1H-benz[f]isoindole-1,3(2H)-dione (17). A solution of $16(8.20 \mathrm{~g}, 34.2 \mathrm{mmol})$ and $N$-methylmaleimide $\mathbf{1}(3.80 \mathrm{~g}, 34.2 \mathrm{mmol})$ in xylene (350 mL) was heated at $140{ }^{\circ} \mathrm{C}$ for $12 \mathrm{~h}$. The solution was allowed to cool to room temperature and was concentrated in vacuo to give adduct $\mathbf{1 7}$ as yellow solid (11.6 g, 97\% yield). An analytical sample of 17 $(83 \mathrm{mg}$ ) was obtained as yellow solid by crystallization of a sample of the above product (200 $\mathrm{mg})$ from a mixture of hexane $(3 \mathrm{~mL})$ and EtOAc $(1 \mathrm{~mL})$. m.p. $168-169{ }^{\circ} \mathrm{C}\left(\right.$ EtOAc/hexane); ${ }^{1} \mathrm{H}-\mathrm{NMR}(400 \mathrm{MHz}$, $\left.\mathrm{CDCl}_{3}\right): \delta=2.50\left(\mathrm{~s}, 3 \mathrm{H}, \mathrm{N}-\mathrm{CH}_{3}\right), 3.31\left(\mathrm{dd},{ }^{3} J_{(\mathrm{H}, \mathrm{H})}=8.4 \mathrm{~Hz},{ }^{3} J_{(\mathrm{H}, \mathrm{H})}=3.2 \mathrm{~Hz}, 1 \mathrm{H}, 9 \mathrm{a}-\mathrm{H}\right), 3.44\left(\mathrm{~d},{ }^{3} J_{(\mathrm{H}, \mathrm{H})}\right.$ $=8.4 \mathrm{~Hz}, 1 \mathrm{H}, 3 \mathrm{a}-\mathrm{H}), 3.82\left(\mathrm{~s}, 3 \mathrm{H}, \mathrm{OCH}_{3}\right), 4.70\left(\mathrm{~d},{ }^{3} J_{(\mathrm{H}, \mathrm{H})}=3.2 \mathrm{~Hz}, 1 \mathrm{H}, 9-\mathrm{H}\right), 5.53\left(\mathrm{~d},{ }^{2} J_{(\mathrm{H}, \mathrm{H})}=5.4 \mathrm{~Hz}\right.$, $1 \mathrm{H})$ and $5.55\left(\mathrm{~d},{ }^{2} J_{(\mathrm{H}, \mathrm{H})}=5.4 \mathrm{~Hz}, 1 \mathrm{H}\right)\left(\mathrm{OCH}_{2} \mathrm{O}\right), 7.12\left(\right.$ pseudo dt, ${ }^{4} J_{(\mathrm{H}, \mathrm{H})}=1.2 \mathrm{~Hz},{ }^{3} J_{(\mathrm{H}, \mathrm{H})}=7.4 \mathrm{~Hz}, 1 \mathrm{H}$, 7-H), 7.17 (overlapped pseudo dt, ${ }^{4} J_{(\mathrm{H}, \mathrm{H})}=1.2 \mathrm{~Hz},{ }^{3} J_{(\mathrm{H}, \mathrm{H})}=7.2 \mathrm{~Hz}, 1 \mathrm{H}, 6-\mathrm{H}$ ), 7.20 (overlapped pseudo $\left.\mathrm{dt},{ }^{4} J_{(\mathrm{H}, \mathrm{H})}=1.2 \mathrm{~Hz},{ }^{3} J_{(\mathrm{H}, \mathrm{H})}=7.3 \mathrm{~Hz}, 1 \mathrm{H}, 12-\mathrm{H}\right), 7.21-7.26$ (complex signal, $2 \mathrm{H}, 8-\mathrm{H}$ and 13-H), 7.38 $\left(\mathrm{dd},{ }^{3} J_{(\mathrm{H}, \mathrm{H})}=7.4 \mathrm{~Hz},{ }^{4} J_{(\mathrm{H}, \mathrm{H})}=1.4 \mathrm{~Hz}, 1 \mathrm{H}, 11-\mathrm{H}\right), 7.65\left(\mathrm{dd},{ }^{3} J_{(\mathrm{H}, \mathrm{H})}=7.2 \mathrm{~Hz},{ }^{4} J_{(\mathrm{H}, \mathrm{H})}=1.2 \mathrm{~Hz}, 1 \mathrm{H}, 5-\mathrm{H}\right)$, $7.68 \mathrm{ppm}\left(\mathrm{dd},{ }^{3} J_{(\mathrm{H}, \mathrm{H})}=7.6 \mathrm{~Hz},{ }^{4} J_{(\mathrm{H}, \mathrm{H})}=1.2 \mathrm{~Hz}, 1 \mathrm{H}, 14-\mathrm{H}\right) ;{ }^{13} \mathrm{C}-\mathrm{NMR}\left(100.6 \mathrm{MHz}, \mathrm{CDCl}_{3}\right): \delta=24.3$ $\left(\mathrm{CH}_{3}, \mathrm{~N}-\mathrm{CH}_{3}\right), 44.6(\mathrm{CH}, \mathrm{C} 9), 47.4(\mathrm{CH}, \mathrm{C} 3 \mathrm{a}), 47.9(\mathrm{CH}, \mathrm{C} 9 \mathrm{a}), 57.1\left(\mathrm{CH}_{3}, \mathrm{OCH}_{3}\right), 81.8(\mathrm{C}, \mathrm{C} 4), 93.8\left(\mathrm{CH}_{2}\right.$, $\left.\mathrm{OCH}_{2} \mathrm{O}\right), 121.8(\mathrm{CH}, \mathrm{C} 5), 122.2(\mathrm{CH}, \mathrm{C} 14), 124.0(\mathrm{CH}, \mathrm{C} 11), 124.4(\mathrm{CH}, \mathrm{C} 8), 126.6(\mathrm{CH}, \mathrm{C} 13), 126.9(\mathrm{CH}$, C12), 127.00 (CH, C7), 127.03 (CH, C6), 136.2 (C, C8a), 139.7 (C, C4a), 140.2 (C, C10), 141.6 (C, C15), 174.1 (C, C1), 176.2 ppm (C, C3); IR (ATR): ̌ = 1692 (s), 1457 (m), 1430 (m), 1292 (m), 1276 (m) 1163 (m), $1124(\mathrm{~m}), 1050(\mathrm{~m}), 1013(\mathrm{~m}), 963(\mathrm{~m}), 771(\mathrm{~m}), 738 \mathrm{~cm}^{-1}(\mathrm{~s})$; accurate mass measurement: $\mathrm{m} / \mathrm{z}$ calcd. for $\mathrm{C}_{21} \mathrm{H}_{19} \mathrm{NO}_{4}+\mathrm{Na}^{+}$: 372.1206; found: 372.1198; elemental analysis calcd. (\%) for $\mathrm{C}_{21} \mathrm{H}_{19} \mathrm{NO}_{4}$ : $\mathrm{C}$ 72.19, H 5.48, N 4.01; found: C 72.08, H 5.42, N 3.79.

4-[(Methoxy)methoxy)-2-methyl-17-methylene-4,9-dihydro-4,9[1',2']benzeno-3a,9a-propano-1H-benz[f]isoindole1,3(2H)-dione (18). A solution of $n$-BuLi in hexanes $(2.75 \mathrm{~mL}, 2.5 \mathrm{M}, 6.87 \mathrm{mmol})$ was added dropwise to a cold $\left(-78{ }^{\circ} \mathrm{C}\right.$, acetone/solid $\mathrm{CO}_{2}$ bath) and magnetically-stirred solution of diisopropylamine $(0.97 \mathrm{~mL}, 6.9 \mathrm{mmol})$ in anhydrous THF $(18 \mathrm{~mL})$ under an Ar atmosphere. When $n$-BuLi addition was finished, the solution was allowed to warm to $0{ }^{\circ} \mathrm{C}$ for $1 \mathrm{~h}$, it was cooled again to $-78{ }^{\circ} \mathrm{C}$, and a solution of $17(1.00 \mathrm{~g}, 2.86 \mathrm{mmol})$ in anhydrous THF (18 mL) was added dropwise. Then, the solution was stirred a $-78{ }^{\circ} \mathrm{C}$ for $15 \mathrm{~min}$ and allowed to warm to $0{ }^{\circ} \mathrm{C}$ for $1 \mathrm{~h}$. The solution was

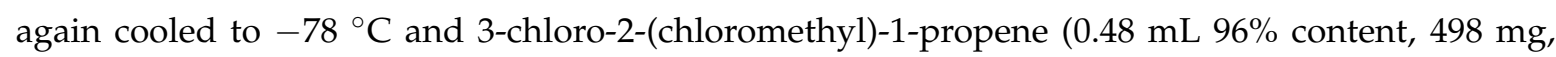
$3.98 \mathrm{mmol}$ ) was added dropwise. The reaction mixture was allowed to warm to room temperature and it was stirred for three days at this temperature. The mixture was made acidic with aqueous $2 \mathrm{~N} \mathrm{HCl}(8 \mathrm{~mL})$ and was extracted with $\mathrm{Et}_{2} \mathrm{O}(3 \times 40 \mathrm{~mL})$. The combined organic extracts were dried (anhydrous $\mathrm{Na}_{2} \mathrm{SO}_{4}$ ) and concentrated in vacuo to give a brown waxy residue (1.29 $\left.\mathrm{g}\right)$ that was subjected to column chromatography (silica gel 35-70 $\mu \mathrm{m}, 65 \mathrm{~g}$, hexane/EtOAc mixtures) to 
give an elution with hexane/EtOAc 19:1 to 9:1, product 18 (801 $\mathrm{mg}, 70 \%$ yield) as yellow solid. m.p 150-152 ${ }^{\circ} \mathrm{C}$ (EtOAc/hexane); ${ }^{1} \mathrm{H}-\mathrm{NMR}\left(400 \mathrm{MHz}, \mathrm{CDCl}_{3}\right): \delta=1.99-2.04\left(\mathrm{dm},{ }^{2} \mathrm{~J}_{(\mathrm{H}, \mathrm{H})}=15.2 \mathrm{~Hz}, 1 \mathrm{H}\right.$, $\left.16-\mathrm{H}_{\mathrm{n}}\right), 2.25-2.31\left(\mathrm{dm},{ }^{2} J_{(\mathrm{H}, \mathrm{H})}=15.8 \mathrm{~Hz}, 1 \mathrm{H}, 18-\mathrm{H}_{\mathrm{n}}\right), 2.45\left(\mathrm{~s}, 3 \mathrm{H}, \mathrm{N}-\mathrm{CH}_{3}\right), 2.59\left(\right.$ broad d, ${ }^{2} J_{(\mathrm{H}, \mathrm{H})}=$ $\left.15.2 \mathrm{~Hz}, 1 \mathrm{H}, 16-\mathrm{H}_{\mathrm{x}}\right), 2.68\left(\right.$ broad d $\left.^{2} J_{(\mathrm{H}, \mathrm{H})}=15.8 \mathrm{~Hz}, 1 \mathrm{H}, 18-\mathrm{H}_{\mathrm{x}}\right), 3.80\left(\mathrm{~s}, 3 \mathrm{H}, \mathrm{OCH}_{3}\right), 4.44(\mathrm{~s}, 1 \mathrm{H}, 9-\mathrm{H})$, $4.58-4.60\left(\mathrm{~m}, 1 \mathrm{H}, 17=\mathrm{CH}_{\mathrm{a}}\right), 4.60-4.62\left(\mathrm{~m}, 1 \mathrm{H}, 17=\mathrm{CH}_{\mathrm{s}}\right), 5.37\left(\mathrm{~d},{ }^{2} J_{(\mathrm{H}, \mathrm{H})}=5.6 \mathrm{~Hz}, 1 \mathrm{H}, \mathrm{OCH}_{\mathrm{a}} \mathrm{O}\right), 5.40$ $\left(\mathrm{d},{ }^{2} J_{(\mathrm{H}, \mathrm{H})}=5.6 \mathrm{~Hz}, 1 \mathrm{H}, \mathrm{OCH} \mathrm{b}_{\mathrm{b}} \mathrm{O}\right), 7.13$ (pseudo dt, $\left.{ }^{4} J_{(\mathrm{H}, \mathrm{H})}=1.4 \mathrm{~Hz},{ }^{3} J_{(\mathrm{H}, \mathrm{H})}=7.2 \mathrm{~Hz}, 1 \mathrm{H}, 7-\mathrm{H}\right), 7.18$ (pseudo dt, $\left.{ }^{4} J_{(\mathrm{H}, \mathrm{H})}=1.5 \mathrm{~Hz},{ }^{3} J_{(\mathrm{H}, \mathrm{H})}=7.6 \mathrm{~Hz}, 1 \mathrm{H}, 6-\mathrm{H}\right), 7.22-7.27$ (complex signal, 2H, 8-H, 12-H), 7.28 (overlapped pseudo dt, $\left.{ }^{4} J_{(\mathrm{H}, \mathrm{H})}=1.6 \mathrm{~Hz},{ }^{3} J_{(\mathrm{H}, \mathrm{H})}=7.2 \mathrm{~Hz}, 1 \mathrm{H}, 13-\mathrm{H}\right), 7.37-7.39\left(\mathrm{ddm},{ }^{3} J_{(\mathrm{H}, \mathrm{H})}=7.2 \mathrm{~Hz}\right.$, $\left.{ }^{4} J_{(\mathrm{H}, \mathrm{H})}=1.6 \mathrm{~Hz}, 1 \mathrm{H}, 11-\mathrm{H}\right), 7.74-7.77 \mathrm{ppm}\left(\right.$ broad d, ${ }^{3} J_{(\mathrm{H}, \mathrm{H})}=7.6 \mathrm{~Hz}, 2 \mathrm{H}, 5-\mathrm{H}$ and $\left.14-\mathrm{H}\right) ;{ }^{13} \mathrm{C}-\mathrm{NMR}$ $\left(100.6 \mathrm{MHz}, \mathrm{CDCl}_{3}\right): \delta=24.6\left(\mathrm{CH}_{3}, \mathrm{~N}-\mathrm{CH}_{3}\right), 38.2\left(\mathrm{CH}_{2}, \mathrm{C} 18\right), 39.0\left(\mathrm{CH}_{2}, \mathrm{C} 16\right), 48.7(\mathrm{CH}, \mathrm{C} 9), 56.4\left(\mathrm{CH}_{3}\right.$, $\left.\mathrm{OCH}_{3}\right), 64.1(\mathrm{C}, \mathrm{C} 3 \mathrm{a}), 64.8(\mathrm{C}, \mathrm{C} 9 \mathrm{a}), 87.1(\mathrm{C}, \mathrm{C} 4), 94.2\left(\mathrm{CH}_{2}, \mathrm{OCH}_{2} \mathrm{O}\right), 108.9\left(\mathrm{CH}_{2}, 17=\mathrm{CH}_{2}\right), 122.7(\mathrm{CH}$, C5), $124.3(\mathrm{CH}, \mathrm{C} 14), 124.8(\mathrm{CH}, \mathrm{C} 8), 126.3(\mathrm{CH}, \mathrm{C} 11), 126.9(\mathrm{CH}, \mathrm{C} 13), 127.0(\mathrm{CH}, \mathrm{C} 6), 127.20(\mathrm{CH})$ and 127.21 (CH) (C7 and C12), 138.15 (C, C8a), 138.21 (C, C10), 139.4 (C, C4a), 139.9 (C, C15), 146.6 (C, C17), 177.2 (C, C3), 179.4 ppm (C, C1); IR (ATR): $\check{v}=1696$ (s), 1456 (m), 1428 (m), 1373 (m), 1304 (m) 1158 (m), $1092(\mathrm{~m}), 1020(\mathrm{~m}), 987(\mathrm{~m}), 930(\mathrm{~m}), 773(\mathrm{~m}), 758 \mathrm{~cm}^{-1}(\mathrm{~m})$; accurate mass measurement: $\mathrm{m} / z$ calcd. for $\mathrm{C}_{25} \mathrm{H}_{23} \mathrm{NO}_{4}+\mathrm{Na}^{+}$: 424.1519. found: 424.1521 ; elemental analysis calcd. (\%) for $\mathrm{C}_{25} \mathrm{H}_{23} \mathrm{NO}_{4}$ : C 74.80, H 5.77, N 3.49; found: C 74.92, H 5.74, N 3.39.

Syn-17-(hydroxymethyl)-4-(methoxymethoxy)-2-methyl-4,9-dihydro-4,9[1' $\left.2^{\prime}\right]$ benzeno-3a,9a-propano-1Hbenz[f]isoindole-1,3(2H)-dione (19) and anti-17-(hydroxymethyl)-4-(methoxymethoxy)-2-methyl-4,9-dihydro4,9[1',2']benzeno-3a,9a-propano-1H-benz[flisoindole-1,3(2H)-dione (20). A solution of the $\mathrm{BH}_{3} \mathrm{THF}$ complex in anhydrous THF $(26.5 \mathrm{~mL}, 1 \mathrm{M}$ in THF, $26.5 \mathrm{mmol})$ was added dropwise to a cold $\left(0{ }^{\circ} \mathrm{C}\right.$, ice-water bath) and magnetically-stirred solution of compound $18(4.62 \mathrm{~g}, 11.5 \mathrm{mmol})$ in anhydrous THF $(150 \mathrm{~mL})$ under an Ar atmosphere, and the reaction mixture was stirred at $0{ }^{\circ} \mathrm{C}$ for $4 \mathrm{~h}$. After addition of $\mathrm{EtOH}(12.7 \mathrm{~mL})$, the mixture was allowed to warm to room temperature, and aqueous solutions of $35 \% \mathrm{H}_{2} \mathrm{O}_{2}(9.4 \mathrm{~mL})$ and $3 \mathrm{M} \mathrm{NaOH}(14.8 \mathrm{~mL})$ were simultaneously added dropwise in $15 \mathrm{~min}$, occasionally cooling with a water bath, and the reaction mixture was stirred at room temperature for $15 \mathrm{~min}$. Water $(50 \mathrm{~mL})$ and EtOAc $(100 \mathrm{~mL})$ were added, the organic phase was separated and the aqueous one was extracted with EtOAc $(2 \times 100 \mathrm{~mL})$. The combined organic phases were dried (anhydrous $\mathrm{Na}_{2} \mathrm{SO}_{4}$ ) and concentrated to dryness in vacuo to give a white solid ( $5.5 \mathrm{~g}$ ) that was subjected to column chromatography (silica gel 35-70 $\mu \mathrm{m}, 165 \mathrm{~g}$, hexane/EtOAc mixtures). Alcohol $19(1.40 \mathrm{~g})$, a 2:1 mixture of 19 and $20(1.174 \mathrm{~g})$ and alcohol $20(330 \mathrm{mg})$ were successively eluted as white solids with hexane/EtOAc 3:2, 1:1, and 2:3, respectively (overall yield: $2.56 \mathrm{~g}$, 72\%, approximate ratio $19 / 20=2.8 / 1\left({ }^{1} \mathrm{H}-\mathrm{NMR}\right)$.

Analytical and spectroscopic data of 19. White solid, m.p. $216-222{ }^{\circ} \mathrm{C}(\mathrm{EtOAc} / \mathrm{hexane}) ;{ }^{1} \mathrm{H}-\mathrm{NMR}$ $\left(400 \mathrm{MHz}, \mathrm{CDCl}_{3}\right): \delta=0.92\left(\mathrm{t},{ }^{2} J_{(\mathrm{H}, \mathrm{H})}={ }^{3} J_{(\mathrm{H}, \mathrm{H})}=12.8 \mathrm{~Hz}, 1 \mathrm{H}, 16-\mathrm{H}_{\mathrm{n}}\right), 1.14\left(\mathrm{t},{ }^{2} J_{(\mathrm{H}, \mathrm{H})}={ }^{3} J_{(\mathrm{H}, \mathrm{H})}=12.8 \mathrm{~Hz}\right.$, $\left.1 \mathrm{H}, 18-\mathrm{H}_{\mathrm{n}}\right), 1.20-1.34$ (broad s, OH), $1.84-1.97(\mathrm{~m}, 1 \mathrm{H}, 17-\mathrm{H}), 2.16\left(\mathrm{ddd},{ }^{2} J_{(\mathrm{H}, \mathrm{H})}=12.8 \mathrm{~Hz},{ }^{3} J_{(\mathrm{H}, \mathrm{H})}=\right.$ $\left.6.0 \mathrm{~Hz},{ }^{4} J_{(\mathrm{H}, \mathrm{H})}=1.6 \mathrm{~Hz}, 1 \mathrm{H}, 16-\mathrm{H}_{\mathrm{x}}\right), 2.28\left(\mathrm{ddd},{ }^{2} J_{(\mathrm{H}, \mathrm{H})}=12.8 \mathrm{~Hz},{ }^{3} J_{(\mathrm{H}, \mathrm{H})}=6.0 \mathrm{~Hz},{ }^{4} J_{(\mathrm{H}, \mathrm{H})}=1.2 \mathrm{~Hz}, 1 \mathrm{H}\right.$, $\left.18-\mathrm{H}_{\mathrm{x}}\right), 2.46\left(\mathrm{~s}, 3 \mathrm{H}, \mathrm{N}-\mathrm{CH}_{3}\right), 3.20\left(\mathrm{dd},{ }^{2} J_{(\mathrm{H}, \mathrm{H})}=11.2 \mathrm{~Hz},{ }^{3} J_{(\mathrm{H}, \mathrm{H})}=5.6 \mathrm{~Hz}, 1 \mathrm{H}, \mathrm{CH}_{a} \mathrm{OH}\right), 3.23\left(\mathrm{dd},{ }^{2} J_{(\mathrm{H}, \mathrm{H})}=\right.$ $\left.11.2 \mathrm{~Hz},{ }^{3} J_{(\mathrm{H}, \mathrm{H})}=5.6 \mathrm{~Hz}, 1 \mathrm{H}, \mathrm{CH}_{b} \mathrm{OH}\right), 3.78\left(\mathrm{~s}, 3 \mathrm{H}, \mathrm{OCH}_{3}\right), 4.40(\mathrm{~s}, 1 \mathrm{H}, 9-\mathrm{H}), 5.36\left(\mathrm{~d},{ }^{2} J_{(\mathrm{H}, \mathrm{H})}=5.6 \mathrm{~Hz}, 1 \mathrm{H}\right.$, $\left.\mathrm{OCH}_{\mathrm{a}} \mathrm{O}\right), 5.39\left(\mathrm{~d},{ }^{2} J_{(\mathrm{H}, \mathrm{H})}=5.6 \mathrm{~Hz}, 1 \mathrm{H}, \mathrm{OCH}_{\mathrm{b}} \mathrm{O}\right), 7.11$ (pseudo dt, ${ }^{4} J_{(\mathrm{H}, \mathrm{H})}=1.6 \mathrm{~Hz},{ }^{3} J_{(\mathrm{H}, \mathrm{H})}=7.2 \mathrm{~Hz}, 1 \mathrm{H}$, 7-H), 7.16 (pseudo dt, $\left.{ }^{4} J_{(\mathrm{H}, \mathrm{H})}=1.6 \mathrm{~Hz},{ }^{3} J_{(\mathrm{H}, \mathrm{H})}=7.4 \mathrm{~Hz}, 1 \mathrm{H}, 6-\mathrm{H}\right), 7.21$ (overlapped dd, ${ }^{3} J_{(\mathrm{H}, \mathrm{H})}=7.2 \mathrm{~Hz}$, $\left.{ }^{4} J_{(\mathrm{H}, \mathrm{H})}=1.6 \mathrm{~Hz}, 1 \mathrm{H}, 8-\mathrm{H}\right), 7.24$ (overlapped pseudo dt, $\left.{ }^{4} J_{(\mathrm{H}, \mathrm{H})}=1.2 \mathrm{~Hz},{ }^{3} J_{(\mathrm{H}, \mathrm{H})}=6.8 \mathrm{~Hz}, 1 \mathrm{H}, 12-\mathrm{H}\right), 7.28$ (overlapped pseudo dt, $\left.{ }^{4} J_{(\mathrm{H}, \mathrm{H})}=1.4 \mathrm{~Hz},{ }^{3} J_{(\mathrm{H}, \mathrm{H})}=7.3 \mathrm{~Hz}, 1 \mathrm{H}, 13-\mathrm{H}\right), 7.37\left(\mathrm{dd},{ }^{3} J_{(\mathrm{H}, \mathrm{H})}=6.6 \mathrm{~Hz},{ }^{4} J_{(\mathrm{H}, \mathrm{H})}=\right.$ $1.4 \mathrm{~Hz}, 1 \mathrm{H}, 11-\mathrm{H}), 7.70-7.73\left(\mathrm{ddm},{ }^{3} J_{(\mathrm{H}, \mathrm{H})}=7.4 \mathrm{~Hz},{ }^{4} J_{(\mathrm{H}, \mathrm{H})}=1.6 \mathrm{~Hz}, 1 \mathrm{H}, 5-\mathrm{H}\right), 7.75-7.78 \mathrm{ppm}(\mathrm{ddm}$, $\left.{ }^{3} J_{(\mathrm{H}, \mathrm{H})}=7.6 \mathrm{~Hz},{ }^{4} J_{(\mathrm{H}, \mathrm{H})}=1.6 \mathrm{~Hz}, 1 \mathrm{H}, 14-\mathrm{H}\right) ;{ }^{13} \mathrm{C}-\mathrm{NMR}\left(100.6 \mathrm{MHz}, \mathrm{CDCl}_{3}\right): \delta=24.6\left(\mathrm{CH}_{3}, \mathrm{~N}-\mathrm{CH}_{3}\right)$, $34.4\left(\mathrm{CH}_{2}, \mathrm{C} 18\right), 35.0\left(\mathrm{CH}_{2}, \mathrm{C} 16\right), 42.5(\mathrm{CH}, \mathrm{C} 17), 48.6(\mathrm{CH}, \mathrm{C} 9), 56.4\left(\mathrm{CH}_{3}, \mathrm{OCH}_{3}\right), 64.9(\mathrm{C}, \mathrm{C} 9 \mathrm{a}), 65.0$ $\left(\mathrm{CH}_{2}, \mathrm{CH}_{2} \mathrm{OH}\right), 65.4(\mathrm{C}, \mathrm{C} 3 \mathrm{a}), 87.0(\mathrm{C}, \mathrm{C} 4), 94.1\left(\mathrm{CH}_{2}, \mathrm{OCH}_{2} \mathrm{O}\right), 122.7(\mathrm{CH}, \mathrm{C} 5), 124.3(\mathrm{CH}, \mathrm{C} 14), 124.7$ $(\mathrm{CH}, \mathrm{C} 8), 126.4(\mathrm{CH}, \mathrm{C} 11), 126.7(\mathrm{CH}, \mathrm{C} 13), 126.9(\mathrm{CH}, \mathrm{C} 6), 127.1(2 \mathrm{CH}, \mathrm{C} 7$ and $\mathrm{C} 12), 138.3(\mathrm{C}, \mathrm{C} 8 \mathrm{a})$, 
138.4 (C, C10), 139.6 (C, C4a), 139.9 (C, C15), 177.5 (C, C3), 179.7 ppm (C, C1); IR (ATR): $\check{v}=3457$ (m), $1767(\mathrm{w}), 1685(\mathrm{~s}), 1433(\mathrm{~m}), 1378(\mathrm{~m}), 1299(\mathrm{~m}), 1165(\mathrm{~m}), 1145(\mathrm{~m}), 1072(\mathrm{~m}), 1053(\mathrm{~s}), 1004(\mathrm{~s}), 794(\mathrm{~m})$, $760 \mathrm{~cm}^{-1}$ (s); accurate mass measurement: $\mathrm{m} / z$ calcd. for $\mathrm{C}_{25} \mathrm{H}_{25} \mathrm{NO}_{5}+\mathrm{Na}^{+} 442.1625$; found: 442.1616 ; elemental analysis calcd. (\%) for $\mathrm{C}_{25} \mathrm{H}_{25} \mathrm{NO}_{5}$ : C 71.58, H 6.01, N 3.34; found: C 71.87, H 5.99, N 3.24.

Analytical and spectroscopic data of 20. White solid, m.p. $186-187^{\circ} \mathrm{C}$ (EtOAc/hexane); ${ }^{1} \mathrm{H}-\mathrm{NMR}$ $\left(400 \mathrm{MHz}, \mathrm{CDCl}_{3}\right): \delta=1.09-1.20(\mathrm{~m}, 1 \mathrm{H}, 17-\mathrm{H}), 1.27-1.35($ broad s, $1 \mathrm{H}, \mathrm{OH}), 1.71\left(\mathrm{dd},{ }^{2} J_{(\mathrm{H}, \mathrm{H})}=14.0 \mathrm{~Hz}\right.$, $\left.{ }^{3} J_{(\mathrm{H}, \mathrm{H})}=7.6 \mathrm{~Hz}, 1 \mathrm{H}, 16-\mathrm{H}_{\mathrm{n}}\right), 1.79-1.85$ (overlapped dd, ${ }^{2} J_{(\mathrm{H}, \mathrm{H})}=14.2 \mathrm{~Hz},{ }^{3} J_{(\mathrm{H}, \mathrm{H})}=6.8 \mathrm{~Hz}, 1 \mathrm{H}, 18-\mathrm{H}_{\mathrm{x}}$ ), 1.83-1.89 (overlapped dd, $\left.{ }^{2} J_{(\mathrm{H}, \mathrm{H})}=14.0 \mathrm{~Hz},{ }^{3} J_{(\mathrm{H}, \mathrm{H})}=6.8 \mathrm{~Hz}, 1 \mathrm{H}, 16-\mathrm{H}_{\mathrm{x}}\right), 2.12\left(\mathrm{dd},{ }^{2} J_{(\mathrm{H}, \mathrm{H})}=14.2 \mathrm{~Hz}\right.$, $\left.{ }^{3} J_{(\mathrm{H}, \mathrm{H})}=7.8 \mathrm{~Hz}, 1 \mathrm{H}, 18-\mathrm{H}_{\mathrm{n}}\right), 2.41\left(\mathrm{~s}, 3 \mathrm{H}, \mathrm{N}-\mathrm{CH}_{3}\right), 3.19\left(\right.$ broad d, $\left.{ }^{3} J_{(\mathrm{H}, \mathrm{H})}=5.2 \mathrm{~Hz}, 2 \mathrm{H}, \mathrm{CH}_{2} \mathrm{OH}\right), 3.79$ $\left(\mathrm{s}, 3 \mathrm{H}, \mathrm{OCH}_{3}\right), 4.43(\mathrm{~s}, 1 \mathrm{H}, 9-\mathrm{H}), 5.36\left(\mathrm{~d},{ }^{2} J_{(\mathrm{H}, \mathrm{H})}=5.8 \mathrm{~Hz}, 1 \mathrm{H}\right)$ and $5.39\left(\mathrm{~d},{ }^{2} J_{(\mathrm{H}, \mathrm{H})}=5.8 \mathrm{~Hz}\right)\left(\mathrm{OCH}_{2} \mathrm{O}\right)$, 7.11-7.16 (overlapped pseudo dt, $\left.{ }^{4} J_{(\mathrm{H}, \mathrm{H})}=1.2 \mathrm{~Hz},{ }^{3} J_{(\mathrm{H}, \mathrm{H})}=7.6 \mathrm{~Hz}, 1 \mathrm{H}, 7-\mathrm{H}\right), 7.14-7.19$ (overlapped pseudo dt, $\left.{ }^{4} J_{(\mathrm{H}, \mathrm{H})}=1.4 \mathrm{~Hz},{ }^{3} J_{(\mathrm{H}, \mathrm{H})}=7.6 \mathrm{~Hz}, 1 \mathrm{H}, 6-\mathrm{H}\right), 7.22-7.24\left(\right.$ overlapped dd, ${ }^{4} J_{(\mathrm{H}, \mathrm{H})}=1.2 \mathrm{~Hz},{ }^{3} J_{(\mathrm{H}, \mathrm{H})}$ $=7.0 \mathrm{~Hz}, 1 \mathrm{H}, 8-\mathrm{H}), 7.24-7.28$ (overlapped pseudo dt, $\left.{ }^{4} J_{(\mathrm{H}, \mathrm{H})}=1.2 \mathrm{~Hz},{ }^{3} J_{(\mathrm{H}, \mathrm{H})}=7.6 \mathrm{~Hz}, 1 \mathrm{H}, 12-\mathrm{H}\right)$, $7.28-7.32$ (overlapped pseudo dt, $\left.{ }^{4} J_{(\mathrm{H}, \mathrm{H})}=1.6 \mathrm{~Hz},{ }^{3} J_{(\mathrm{H}, \mathrm{H})}=8.0 \mathrm{~Hz}, 1 \mathrm{H}, 13-\mathrm{H}\right), 7.39\left(\mathrm{dd}, 4 J_{(\mathrm{H}, \mathrm{H})}=1.4 \mathrm{~Hz}\right.$, $\left.{ }^{3} J_{(\mathrm{H}, \mathrm{H})}=7.4 \mathrm{~Hz}, 1 \mathrm{H}, 11-\mathrm{H}\right), 7.74\left(\mathrm{dd},{ }^{4} J_{(\mathrm{H}, \mathrm{H})}=1.6 \mathrm{~Hz},{ }^{3} J_{(\mathrm{H}, \mathrm{H})}=7.2 \mathrm{~Hz}, 1 \mathrm{H}, 5-\mathrm{H}\right), 7.79 \mathrm{ppm}\left(\mathrm{dd},{ }^{4} J_{(\mathrm{H}, \mathrm{H})}\right.$ $\left.=1.2 \mathrm{~Hz},{ }^{3} J_{(\mathrm{H}, \mathrm{H})}=7.6 \mathrm{~Hz}, 1 \mathrm{H}, 14-\mathrm{H}\right) ;{ }^{13} \mathrm{C}-\mathrm{NMR}\left(100.6 \mathrm{MHz}, \mathrm{CDCl}_{3}\right): \delta=24.4\left(\mathrm{CH}_{3}, \mathrm{~N}-\mathrm{CH}_{3}\right), 33.0$ $\left(\mathrm{CH}_{2}, \mathrm{C} 18\right), 35.2\left(\mathrm{CH}_{2}, \mathrm{C} 16\right), 43.5(\mathrm{CH}, \mathrm{C} 17), 49.9(\mathrm{CH}, \mathrm{C} 9), 56.4\left(\mathrm{CH}_{3}, \mathrm{OCH}_{3}\right), 65.4(\mathrm{C}, \mathrm{C} 9), 65.5\left(\mathrm{CH}_{2}\right.$, $\left.\mathrm{CH}_{2} \mathrm{OH}\right), 66.5(\mathrm{C}, \mathrm{C} 3 \mathrm{a}), 87.5(\mathrm{C}, \mathrm{C} 4), 94.4\left(\mathrm{CH}_{2}, \mathrm{OCH}_{2} \mathrm{O}\right), 122.7(\mathrm{CH}, \mathrm{C} 5), 124.0(\mathrm{CH}, \mathrm{C} 14), 124.8(\mathrm{CH}$, C8), 125.8 (CH, C11), 126.9 (2 CH, C6 and C13), 127.2 (CH, C7), 127.3 (CH, C12), 138.3 (C, C10), $138.4(\mathrm{C}$, C8a), 139.1 (C, C4a), 140.0 (C, C15), 177.6 (C, C3), 179.7 ppm (C, C1); IR (ATR): $\check{v}=3471$ (m), 2946 (w), $2935(\mathrm{w}), 2855(\mathrm{w}), 1768(\mathrm{w}), 1691(\mathrm{~s}), 1456(\mathrm{~m}), \mathrm{n} 1434(\mathrm{~m}), 1381(\mathrm{~m}), 1300(\mathrm{~m}), 1162(\mathrm{~m}), 1070(\mathrm{~m}), 1043$ (s), $1014(\mathrm{~m}), 990(\mathrm{~s}), 956(\mathrm{~s}), 929(\mathrm{~m}), 773(\mathrm{~m}), 756(\mathrm{~m}), 720 \mathrm{~cm}^{-1}(\mathrm{~m})$; accurate mass measurement: $\mathrm{m} / \mathrm{z}$ calcd. for $\mathrm{C}_{25} \mathrm{H}_{25} \mathrm{NO}_{5}+\mathrm{H}^{+} 420.1805$; found: 420.1815 ; elemental analysis calcd. (\%) for $\mathrm{C}_{25} \mathrm{H}_{25} \mathrm{NO}_{5}: \mathrm{C}$ 71.58, H 6.01, N 3.34; found: C 71.32, H 5.81, N 3.11.

Syn-4-hydroxy-17-(methanesulfonyloxymethyl)-2-methyl-4,9-dihydro-4,9[1' $\left.2^{\prime}\right]$ benzeno-3a,9a-propano-1Hbenz[ffisoindole-1,3(2H)-dione (21). $\mathrm{MsCl}(60 \mu \mathrm{L}, 86 \mathrm{mg}, 0.75 \mathrm{mmol})$ was added dropwise to a cold solution $\left(0^{\circ} \mathrm{C}\right.$, ice-water bath) of alcohol $19(300 \mathrm{mg}, 0.72 \mathrm{mmol})$ and anhydrous $\mathrm{Et}_{3} \mathrm{~N}(0.23 \mathrm{~mL}, 166 \mathrm{mg}$, $1.65 \mathrm{mmol})$ in anhydrous $\mathrm{CH}_{2} \mathrm{Cl}_{2}(14 \mathrm{~mL})$ under an Ar atmosphere, and the mixture was stirred for $4 \mathrm{~h}$ at $0{ }^{\circ} \mathrm{C}$. Saturated aqueous solution of $\mathrm{NaHCO}_{3}(4 \mathrm{~mL})$ and water $(14 \mathrm{~mL})$ were successively added to the reaction mixture. The organic phase was separated and the aqueous one was extracted with $\mathrm{CH}_{2} \mathrm{Cl}_{2}(2 \times 20 \mathrm{~mL})$. The combined organic phases were successively washed with aqueous $1 \mathrm{~N}$ $\mathrm{HCl}(3 \times 15 \mathrm{~mL})$, water $(20 \mathrm{~mL})$ and brine $(20 \mathrm{~mL})$, dried (anhydrous $\left.\mathrm{Na}_{2} \mathrm{SO}_{4}\right)$ and concentrated to dryness in vacuo to give a white solid ( $370 \mathrm{mg}$ ), that was subjected to column chromatography (silica gel 35-70 $\mu \mathrm{m}, 12 \mathrm{~g}$, hexane/EtOAc mixtures) to give mesylate 21 (223 mg, 68\% yield) as white solid, on elution with hexane/EtOAc 3:2 to 1:1. An analytical sample of $21(65 \mathrm{mg})$ was obtained as white solid by crystallization of a sample of the above product $(120 \mathrm{mg})$ from EtOAc $(8 \mathrm{~mL})$. m.p. $228-232{ }^{\circ} \mathrm{C}$ (EtOAc/hexane); ${ }^{1} \mathrm{H}-\mathrm{NMR}\left(400 \mathrm{MHz}, \mathrm{CDCl}_{3}\right): \delta=1.13\left(\mathrm{t},{ }^{2} J_{(\mathrm{H}, \mathrm{H})}={ }^{3} J_{(\mathrm{H}, \mathrm{H})}=12.6 \mathrm{~Hz}, 1 \mathrm{H}, 16-\mathrm{H}_{\mathrm{n}}\right), 1.23$ $\left(\mathrm{t},{ }^{2} J_{(\mathrm{H}, \mathrm{H})}={ }^{3} J_{(\mathrm{H}, \mathrm{H})}=12.6 \mathrm{~Hz}, 1 \mathrm{H}, 18-\mathrm{H}_{\mathrm{n}}\right), 2.09-2.21(\mathrm{~m}, 1 \mathrm{H}, 17-\mathrm{H}), 2.17-2.23\left(\mathrm{ddm},{ }^{2} J_{(\mathrm{H}, \mathrm{H})}=12.8 \mathrm{~Hz}\right.$, $\left.{ }^{3} J_{(\mathrm{H}, \mathrm{H})}=6.0 \mathrm{~Hz}, 1 \mathrm{H}, 18-\mathrm{H}_{\mathrm{x}}\right), 2.30-2.36\left(\mathrm{ddm},{ }^{2} J_{(\mathrm{H}, \mathrm{H})}=12.8 \mathrm{~Hz},{ }^{3} J_{(\mathrm{H}, \mathrm{H})}=6.0 \mathrm{~Hz}, 1 \mathrm{H}, 16-\mathrm{H}_{\mathrm{x}}\right), 2.50(\mathrm{~s}, 3 \mathrm{H}$, $\left.\mathrm{N}-\mathrm{CH}_{3}\right), 2.85\left(\mathrm{~s}, 3 \mathrm{H}, \mathrm{CH}_{3} \mathrm{SO}_{3}\right), 3.81\left(\mathrm{~d},{ }^{3} J_{(\mathrm{H}, \mathrm{H})}=5.6 \mathrm{~Hz}, 2 \mathrm{H}, \mathrm{CH}_{2} \mathrm{OMs}\right), 4.17(\mathrm{~s}, 1 \mathrm{H}, \mathrm{OH}), 4.43(\mathrm{~s}, 1 \mathrm{H}$, 9-H), 7.11 (pseudo dt, ${ }^{4} J_{(\mathrm{H}, \mathrm{H})}=1.2 \mathrm{~Hz},{ }^{3} J_{(\mathrm{H}, \mathrm{H})}=7.2 \mathrm{~Hz}, 1 \mathrm{H}, 7-\mathrm{H}$ ), 7.18 (pseudo dt, ${ }^{4} J_{(\mathrm{H}, \mathrm{H})}=1.2 \mathrm{~Hz}$, $\left.{ }^{3} J_{(\mathrm{H}, \mathrm{H})}=7.6 \mathrm{~Hz}, 1 \mathrm{H}, 6-\mathrm{H}\right), 7.19-7.21$ (overlapped dm, $\left.{ }^{3} J_{(\mathrm{H}, \mathrm{H})}=7.2 \mathrm{~Hz}, 1 \mathrm{H}, 8-\mathrm{H}\right), 7.20-7.24$ (overlapped pseudo dt, $\left.{ }^{4} J_{(\mathrm{H}, \mathrm{H})}=1.2 \mathrm{~Hz},{ }^{3} J_{(\mathrm{H}, \mathrm{H})}=7.6 \mathrm{~Hz}, 1 \mathrm{H}, 12-\mathrm{H}\right), 7.30$ (pseudo dt, ${ }^{4} J_{(\mathrm{H}, \mathrm{H})}=1.3 \mathrm{~Hz},{ }^{3} J_{(\mathrm{H}, \mathrm{H})}=$ $7.5 \mathrm{~Hz}, 1 \mathrm{H}, 13-\mathrm{H}), 7.36\left(\mathrm{dd},{ }^{3} J_{(\mathrm{H}, \mathrm{H})}=7.2 \mathrm{~Hz},{ }^{4} J_{(\mathrm{H}, \mathrm{H})}=1.2 \mathrm{~Hz}, 1 \mathrm{H}, 11-\mathrm{H}\right), 7.41-7.43\left(\mathrm{dm},{ }^{3} J_{(\mathrm{H}, \mathrm{H})}=7.2 \mathrm{~Hz}\right.$, $1 \mathrm{H}, 5-\mathrm{H}), 7.68-7.71 \mathrm{ppm}\left(\mathrm{dm},{ }^{3} J_{(\mathrm{H}, \mathrm{H})}=7.2 \mathrm{~Hz}, 1 \mathrm{H}, 14-\mathrm{H}\right) ;{ }^{13} \mathrm{C}-\mathrm{NMR}\left(100.6 \mathrm{MHz}, \mathrm{CDCl}_{3}\right): \delta=24.7\left(\mathrm{CH}_{3}\right.$, N-CH $\left.\mathrm{CH}_{3}\right), 33.1\left(\mathrm{CH}_{2}, \mathrm{C} 18\right), 35.2\left(\mathrm{CH}_{2}, \mathrm{C} 16\right), 37.4\left(\mathrm{CH}_{3}, \mathrm{CH}_{3} \mathrm{SO}_{3}\right), 39.2(\mathrm{CH}, \mathrm{C} 17), 48.0(\mathrm{CH}, \mathrm{C} 9), 63.6(\mathrm{C}$, C9a), 63.9 (C, C3a), $69.4\left(\mathrm{CH}_{2}, \mathrm{CH}_{2} \mathrm{OMs}\right), 79.6$ (C, C4), $121.0(\mathrm{CH}, \mathrm{C} 5), 123.1(\mathrm{CH}, \mathrm{C} 14), 124.5$ ( $\left.\mathrm{CH}, \mathrm{C} 8\right)$, $126.1(\mathrm{CH}, \mathrm{C} 11), 126.9(\mathrm{CH}, \mathrm{C} 12), 127.0(2 \mathrm{CH}, \mathrm{C} 6$ and $\mathrm{C} 13), 127.1(\mathrm{CH}, \mathrm{C} 7), 137.6(\mathrm{C}, \mathrm{C} 10), 137.7(\mathrm{C}$, 
C8a), 140.3 (C, C15), 141.4 (C, C4a), 179.5 (C, C1), 180.9 ppm (C, C3); IR (ATR): $\check{v}=3450-3350$ (w, max. at 3498 and 3385), $3028(\mathrm{w}), 2940(\mathrm{w}), 1767(\mathrm{w}), 1684(\mathrm{~s}), 1454(\mathrm{~m}), 1436(\mathrm{~m}), 1351(\mathrm{~s}), 1173(\mathrm{~s}), 1072(\mathrm{~m})$, $1013(\mathrm{~m}), 977(\mathrm{~s}), 956(\mathrm{~s}), 832(\mathrm{~m}), 793(\mathrm{~m}), 754 \mathrm{~cm}^{-1}(\mathrm{~s})$; accurate mass measurement: $m / z$ calcd. for $\mathrm{C}_{24} \mathrm{H}_{23} \mathrm{NO}_{6} \mathrm{~S}+\mathrm{Na}^{+}$: 476.1138; found: 476.1143; elemental analysis calcd. (\%) for $\mathrm{C}_{24} \mathrm{H}_{23} \mathrm{NO}_{6} \mathrm{~S}: \mathrm{C} 63.56$, H 5.11, N 3.09, S 7.07; found: C 63.26, H 5.23, N 2.80, S 6.22.

Syn-17-(methanesulfonyloxymethyl)-4-(metoxymethoxy)-2-methyl-4,9-dihydro-4,9[1',2']benzeno-3a,9a-propano1H-benzlflisoindole-1,3(2H)-dione (22). $\mathrm{MsCl}(0.23 \mathrm{~mL}, 334 \mathrm{mg}, 2.92 \mathrm{mmol})$ was added dropwise to a cold solution $\left(0^{\circ} \mathrm{C}\right.$, ice-water bath) of alcohol $19(1.16 \mathrm{~g}, 2.77 \mathrm{mmol})$ and anhydrous $\mathrm{Et}_{3} \mathrm{~N}(0.89 \mathrm{~mL}$, $646 \mathrm{mg}, 6.4 \mathrm{mmol})$ in anhydrous $\mathrm{CH}_{2} \mathrm{Cl}_{2}(60 \mathrm{~mL})$ under an Ar atmosphere and the mixture was stirred for $4 \mathrm{~h}$ at $0{ }^{\circ} \mathrm{C}$. Saturated aqueous solution of $\mathrm{NaHCO}_{3}(15 \mathrm{~mL})$ and water $(30 \mathrm{~mL})$ were successively added to the reaction mixture. The organic phase was separated and the aqueous one was extracted with $\mathrm{CH}_{2} \mathrm{Cl}_{2}(2 \times 30 \mathrm{~mL})$. The combined organic phases were successively washed with aqueous $1 \mathrm{~N}$ $\mathrm{HCl}(3 \times 30 \mathrm{~mL})$, water $(30 \mathrm{~mL})$ and brine $(30 \mathrm{~mL})$, dried (anhydrous $\left.\mathrm{Na}_{2} \mathrm{SO}_{4}\right)$ and concentrated to dryness in vacuo to give a white solid $(1.32 \mathrm{~g})$, that was subjected to column chromatography ( $40 \mathrm{~g}$ neutral $\mathrm{Al}_{2} \mathrm{O}_{3}$, hexane/EtOAc mixtures) to give mesylate $22(1.25 \mathrm{~g}, 91 \%$ yield as a white solid on elution with hexane/EtOAc 4:1 to 7:3. An analytical sample of $22(54 \mathrm{mg})$ was obtained as white solid by crystallization of a sample of the above product $(130 \mathrm{mg})$ from EtOAc $(3 \mathrm{~mL}) . \mathrm{m} . \mathrm{p} .71-76{ }^{\circ} \mathrm{C}(\mathrm{dec})$ (EtOAc); ${ }^{1} \mathrm{H}-\mathrm{NMR}\left(400 \mathrm{MHz}, \mathrm{CDCl}_{3}\right): \delta=0.96\left(\mathrm{t},{ }^{2} \mathrm{~J}_{(\mathrm{H}, \mathrm{H})}={ }^{3} \mathrm{~J}_{(\mathrm{H}, \mathrm{H})}=12.8 \mathrm{~Hz}, 1 \mathrm{H}, 16-\mathrm{H}_{\mathrm{n}}\right), 1.20\left(\mathrm{t},{ }^{2} \mathrm{~J}_{(\mathrm{H}, \mathrm{H})}\right.$ $\left.={ }^{3} \mathrm{~J}_{(\mathrm{H}, \mathrm{H})}=12.8 \mathrm{~Hz}, 1 \mathrm{H}, 18-\mathrm{H}_{\mathrm{n}}\right), 2.03-2.15(\mathrm{~m}, 1 \mathrm{H}, 17-\mathrm{H}), 2.17-2.23\left(\mathrm{ddm},{ }^{2} \mathrm{~J}_{(\mathrm{H}, \mathrm{H})}=12.8 \mathrm{~Hz},{ }^{3} \mathrm{~J}_{(\mathrm{H}, \mathrm{H})}=\right.$ $\left.6.0 \mathrm{~Hz}, 1 \mathrm{H}, 16-\mathrm{H}_{\mathrm{x}}\right), 2.30-2.36\left(\mathrm{ddm},{ }^{2} \mathrm{~J}_{(\mathrm{H}, \mathrm{H})}=12.8 \mathrm{~Hz},{ }^{3} \mathrm{~J}_{(\mathrm{H}, \mathrm{H})}=6.0 \mathrm{~Hz}, 1 \mathrm{H}, 18-\mathrm{H}_{\mathrm{x}}\right), 2.47\left(\mathrm{~s}, 3 \mathrm{H}, \mathrm{N}-\mathrm{CH}_{3}\right)$, $2.85\left(\mathrm{~s}, 3 \mathrm{H}, \mathrm{CH}_{3} \mathrm{SO}_{3}\right), 3.79$ (overlapped $\mathrm{s}, 3 \mathrm{H}, \mathrm{OCH}_{3}$ ), 3.79-3.80 (overlapped d, $2 \mathrm{H}, \mathrm{CH}_{2} \mathrm{OMs}$ ), 4.41 (s, $1 \mathrm{H}, 9-\mathrm{H}), 5.35\left(\mathrm{~d},{ }^{2} \mathrm{~J}_{(\mathrm{H}, \mathrm{H})}=6.0 \mathrm{~Hz}, 1 \mathrm{H}, \mathrm{OCH}_{\mathrm{a}} \mathrm{O}\right), 5.37\left(\mathrm{~d},{ }^{2} \mathrm{~J}_{(\mathrm{H}, \mathrm{H})}=6.0 \mathrm{~Hz}, 1 \mathrm{H}, \mathrm{OCH}_{\mathrm{b}} \mathrm{O}\right), 7.13$ (pseudo dt, $\left.{ }^{4} \mathrm{~J}_{(\mathrm{H}, \mathrm{H})}=1.4 \mathrm{~Hz},{ }^{3} \mathrm{~J}_{(\mathrm{H}, \mathrm{H})}=7.4 \mathrm{~Hz}, 1 \mathrm{H}, 7-\mathrm{H}\right), 7.18$ (pseudo dt, $\left.{ }^{4} \mathrm{~J}_{(\mathrm{H}, \mathrm{H})}=1.5 \mathrm{~Hz},{ }^{3} \mathrm{~J}_{(\mathrm{H}, \mathrm{H})}=7.6 \mathrm{~Hz}, 1 \mathrm{H}, 6-\mathrm{H}\right)$, $7.23\left(\mathrm{dd},{ }^{3} \mathrm{~J}_{(\mathrm{H}, \mathrm{H})}=7.2 \mathrm{~Hz},{ }^{4} \mathrm{~J}_{(\mathrm{H}, \mathrm{H})}=1.4 \mathrm{~Hz}, 1 \mathrm{H}, 8-\mathrm{H}\right), 7.25-7.29\left(\right.$ pseudo dt, ${ }^{4} \mathrm{~J}_{(\mathrm{H}, \mathrm{H})}=1.4 \mathrm{~Hz},{ }^{3} \mathrm{~J}_{(\mathrm{H}, \mathrm{H})}=$ $7.2 \mathrm{~Hz}, 1 \mathrm{H}, 12-\mathrm{H}), 7.29-7.33\left(\right.$ pseudo dt, $\left.{ }^{4} \mathrm{~J}_{(\mathrm{H}, \mathrm{H})}=1.6 \mathrm{~Hz},{ }^{3} \mathrm{~J}_{(\mathrm{H}, \mathrm{H})}=7.2 \mathrm{~Hz}, 1 \mathrm{H}, 13-\mathrm{H}\right), 7.39\left(\mathrm{dd},{ }^{3} \mathrm{~J}_{(\mathrm{H}, \mathrm{H})}=\right.$ $\left.7.2 \mathrm{~Hz},{ }^{4} \mathrm{~J}_{(\mathrm{H}, \mathrm{H})}=1.6 \mathrm{~Hz}, 1 \mathrm{H}, 11-\mathrm{H}\right), 7.74-7.76$ (overlapped dm, $\left.{ }^{3} \mathrm{~J}_{(\mathrm{H}, \mathrm{H})}=7.2 \mathrm{~Hz}, 1 \mathrm{H}, 5-\mathrm{H}\right), 7.76-7.78 \mathrm{ppm}$ (overlapped dm, $\left.{ }^{3} \mathrm{~J}_{(\mathrm{H}, \mathrm{H})}=7.2 \mathrm{~Hz}, 1 \mathrm{H}, 14-\mathrm{H}\right) ;{ }^{13} \mathrm{C}-\mathrm{NMR}\left(100.6 \mathrm{MHz}, \mathrm{CDCl}_{3}\right): \delta=24.7\left(\mathrm{CH}_{3}, \mathrm{~N}_{-} \mathrm{CH}_{3}\right)$, $34.0\left(\mathrm{CH}_{2}, \mathrm{C} 18\right), 34.7\left(\mathrm{CH}_{2}, \mathrm{C} 16\right), 37.4\left(\mathrm{CH}_{3}, \mathrm{CH}_{3} \mathrm{SO}_{3}\right), 39.3(\mathrm{CH}, \mathrm{C} 17), 48.5(\mathrm{CH}, \mathrm{C} 9), 56.4\left(\mathrm{CH}_{3}, \mathrm{OCH}_{3}\right)$, 64.7 (C, C9a), 65.3 (C, C3a), $69.5\left(\mathrm{CH}_{2}, \mathrm{CH}_{2} \mathrm{OMs}\right), 87.3(\mathrm{C}, \mathrm{C} 4), 94.2\left(\mathrm{CH}_{2}, \mathrm{OCH}_{2} \mathrm{O}\right), 122.8$ (CH, C5), $124.4(\mathrm{CH}, \mathrm{C} 14), 124.8(\mathrm{CH}, \mathrm{C} 8), 126.4(\mathrm{CH}, \mathrm{C} 11), 126.9(\mathrm{CH}, \mathrm{C} 13), 127.0(\mathrm{CH}, \mathrm{C} 6), 127.2(\mathrm{CH})$ and 127.3 (CH) (C7 and C12), 138.0 (C, C8a), 138.2 (C, C10), 139.2 (C, C4a), 139.8 (C, C15), 177.0 (C, C3), $179.1 \mathrm{ppm}(\mathrm{C}, \mathrm{C} 1)$; IR (ATR): ̌̌ = $3012(\mathrm{w}), 2959$ (w), 1769 (w), 1693 (s), 1455 (m), 1430 (m), $1378(\mathrm{~m})$, $1343(\mathrm{~s}), 1171(\mathrm{~s}), 1072(\mathrm{~m}), 1050(\mathrm{~m}), 1006(\mathrm{~m}), 987(\mathrm{~s}), 955(\mathrm{~s}), 926(\mathrm{~s}), 825(\mathrm{~s}), 794(\mathrm{~m}), 754 \mathrm{~cm}^{-1}(\mathrm{~s})$; accurate mass measurement: $\mathrm{m} / \mathrm{z}$ calcd. for $\mathrm{C}_{26} \mathrm{H}_{27} \mathrm{NO}_{7} \mathrm{~S}+\mathrm{Na}^{+}$: 520.1400; found: 520.1412; elemental analysis calcd. (\%) for $\mathrm{C}_{26} \mathrm{H}_{27} \mathrm{NO}_{7} \mathrm{~S}$ : C 62.76, $\mathrm{H}$ 5.47, N 2.82, S 6.44; found: C 62.87, H 5.48, N 2.69, S 6.26 .

Syn-17-(iodomethyl)-4-(methoxymethoxy)-2-methyl-4,9-dihydro-4,9[1', $\left.2^{\prime}\right]$ benzeno-3a,9a-propano-1Hbenzlffisoindole-1,3(2H)-dione (23). A magnetically stirred solution of mesylate $22(668 \mathrm{mg}, 1.34 \mathrm{mmol})$ and $\mathrm{NaI}(2.03 \mathrm{~g}, 99 \%, 13.4 \mathrm{mmol})$ in anhydrous acetone $(40 \mathrm{~mL})$ was heated at reflux for $16 \mathrm{~h}$ under an $\mathrm{Ar}$ atmosphere. The mixture was allowed to cool to room temperature, the precipitate was filtered through a pad of Celite ${ }^{\circledR}$, and the solid was washed with EtOAc $(100 \mathrm{~mL})$. The combined filtrate and washings were concentrated in vacuo, and the residue $(860 \mathrm{mg})$ was subjected to column chromatography (30 g 50-200 $\mu \mathrm{m}$ silica gel, hexane/EtOAc mixtures). On elution with hexane/EtOAc 9:1, iodide 23 ( $617 \mathrm{mg}, 87 \%$ yield) was obtained as yellow solid. An analytical sample of 23 (72 mg) was obtained as white solid by crystallization of a sample of the above product $(100 \mathrm{mg})$ from EtOAc $(0.3 \mathrm{~mL})$ and hexane $(0.5 \mathrm{~mL})$. m.p. $176-177^{\circ} \mathrm{C}(\mathrm{EtOAc} / \mathrm{hexane}) ;{ }^{1} \mathrm{H}-\mathrm{NMR}\left(400 \mathrm{MHz}, \mathrm{CDCl}_{3}\right): \delta=$ $0.92\left(\mathrm{t},{ }^{2} J_{(\mathrm{H}, \mathrm{H})}={ }^{3} J_{(\mathrm{H}, \mathrm{H})}=12.8 \mathrm{~Hz}, 1 \mathrm{H}, 16-\mathrm{H}_{\mathrm{n}}\right), 1.15\left(\mathrm{t},{ }^{2} J_{(\mathrm{H}, \mathrm{H})}={ }^{3} J_{(\mathrm{H}, \mathrm{H})}=12.8 \mathrm{~Hz}, 1 \mathrm{H}, 18-\mathrm{H}_{\mathrm{n}}\right), 1.74-1.86$ $(\mathrm{m}, 1 \mathrm{H}, 17-\mathrm{H}), 2.23\left(\mathrm{ddd},{ }^{2} J_{(\mathrm{H}, \mathrm{H})}=13.0 \mathrm{~Hz},{ }^{3} J_{(\mathrm{H}, \mathrm{H})}=6.0 \mathrm{~Hz},{ }^{4} J_{(\mathrm{H}, \mathrm{H})}=1.4 \mathrm{~Hz}, 1 \mathrm{H}, 16-\mathrm{H}_{\mathrm{x}}\right), 2.34(\mathrm{ddd}$, 
$\left.{ }^{2} J_{(\mathrm{H}, \mathrm{H})}=13.2 \mathrm{~Hz},{ }^{3} J_{(\mathrm{H}, \mathrm{H})}=6.0 \mathrm{~Hz},{ }^{4} J_{(\mathrm{H}, \mathrm{H})}=1.4 \mathrm{~Hz}, 1 \mathrm{H}, 18-\mathrm{H}_{\mathrm{x}}\right), 2.47\left(\mathrm{~s}, 3 \mathrm{H}, \mathrm{N}-\mathrm{CH}_{3}\right), 2.81\left(\mathrm{dd},{ }^{2} J_{(\mathrm{H}, \mathrm{H})}=\right.$ $\left.10.0 \mathrm{~Hz},{ }^{3} J_{(\mathrm{H}, \mathrm{H})}=5.6 \mathrm{~Hz}, 1 \mathrm{H}, \mathrm{CH}_{a} \mathrm{I}\right), 2.84\left(\mathrm{dd},{ }^{2} J_{(\mathrm{H}, \mathrm{H})}=10.0 \mathrm{~Hz},{ }^{3} J_{(\mathrm{H}, \mathrm{H})}=5.6 \mathrm{~Hz}, 1 \mathrm{H}, \mathrm{CH}_{b} \mathrm{I}\right), 3.79(\mathrm{~s}, 3 \mathrm{H}$, $\left.\mathrm{OCH}_{3}\right), 4.39(\mathrm{~s}, 1 \mathrm{H}, 9-\mathrm{H}), 5.36\left(\mathrm{~d},{ }^{2} J_{(\mathrm{H}, \mathrm{H})}=6.0 \mathrm{~Hz}, 1 \mathrm{H}, \mathrm{OCH}_{a} \mathrm{O}\right), 5.37\left(\mathrm{~d},{ }^{2} J_{(\mathrm{H}, \mathrm{H})}=6.0 \mathrm{~Hz}, 1 \mathrm{H}, \mathrm{OCH}_{b} \mathrm{O}\right)$, 7.12 (pseudo dt, $\left.{ }^{4} J_{(\mathrm{H}, \mathrm{H})}=1.5 \mathrm{~Hz},{ }^{3} J_{(\mathrm{H}, \mathrm{H})}=7.2 \mathrm{~Hz}, 1 \mathrm{H}, 7-\mathrm{H}\right), 7.17\left(\right.$ pseudo dt, ${ }^{4} J_{(\mathrm{H}, \mathrm{H})}=1.6 \mathrm{~Hz},{ }^{3} J_{(\mathrm{H}, \mathrm{H})}$ $=7.4 \mathrm{~Hz}, 1 \mathrm{H}, 6-\mathrm{H}), 7.22\left(\mathrm{dd},{ }^{3} J_{(\mathrm{H}, \mathrm{H})}=7.2 \mathrm{~Hz},{ }^{4} J_{(\mathrm{H}, \mathrm{H})}=1.6 \mathrm{~Hz}, 1 \mathrm{H}, 8-\mathrm{H}\right), 7.27$ (pseudo dt, ${ }^{4} J_{(\mathrm{H}, \mathrm{H})}=$ $\left.1.4 \mathrm{~Hz},{ }^{3} J_{(\mathrm{H}, \mathrm{H})}=7.2 \mathrm{~Hz}, 1 \mathrm{H}, 12-\mathrm{H}\right), 7.31$ (pseudo dt, $\left.{ }^{4} J_{(\mathrm{H}, \mathrm{H})}=1.6 \mathrm{~Hz},{ }^{3} J_{(\mathrm{H}, \mathrm{H})}=7.6 \mathrm{~Hz}, 1 \mathrm{H}, 13-\mathrm{H}\right), 7.39$ $\left(\mathrm{dd},{ }^{4} J_{(\mathrm{H}, \mathrm{H})}=1.4 \mathrm{~Hz},{ }^{3} J_{(\mathrm{H}, \mathrm{H})}=7.2 \mathrm{~Hz}, 1 \mathrm{H}, 11-\mathrm{H}\right), 7.73-7.76$ (overlapped dm, $\left.{ }^{3} J_{(\mathrm{H}, \mathrm{H})}=7.6 \mathrm{~Hz}, 1 \mathrm{H}, 5-\mathrm{H}\right)$, 7.75-7.77 ppm (overlapped dm, $\left.{ }^{3} J_{(\mathrm{H}, \mathrm{H})}=7.6 \mathrm{~Hz}, 1 \mathrm{H}, 14-\mathrm{H}\right) ;{ }^{13} \mathrm{C}-\mathrm{NMR}\left(100.6 \mathrm{MHz}, \mathrm{CDCl}_{3}\right): \delta=8.4$ $\left(\mathrm{CH}_{2}, \mathrm{CH}_{2} \mathrm{I}\right), 24.7\left(\mathrm{CH}_{3}, \mathrm{~N}-\mathrm{CH}_{3}\right), 38.0\left(\mathrm{CH}_{2}, \mathrm{C} 18\right), 38.7\left(\mathrm{CH}_{2}, \mathrm{C} 16\right), 41.5(\mathrm{CH}, \mathrm{C} 17), 48.6(\mathrm{CH}, \mathrm{C} 9), 56.4$ $\left(\mathrm{CH}_{3}, \mathrm{OCH}_{3}\right), 64.8(\mathrm{C}, \mathrm{C} 9 \mathrm{a}), 65.6(\mathrm{C}, \mathrm{C} 3 \mathrm{a}), 87.3(\mathrm{C}, \mathrm{C} 4), 94.1\left(\mathrm{CH}_{2}, \mathrm{OCH}_{2} \mathrm{O}\right), 122.8(\mathrm{CH}, \mathrm{C} 5), 124.4(\mathrm{CH}$, C14), $124.8(\mathrm{CH}, \mathrm{C} 8), 126.4(\mathrm{CH}, \mathrm{C} 11), 127.0(\mathrm{CH}, \mathrm{C} 6$ and $\mathrm{C} 13), 127.2(\mathrm{CH}, \mathrm{C} 7), 127.3(\mathrm{CH}, \mathrm{C} 12), 138.1$ (C, C8a), 138.2 (C, C10), 139.3 (C, C4a), 139.8 (C, C15), 177.2 (C, C3), 179.3 ppm (C, C1); IR (ATR): $\check{v}=$ 2936 (w), 1769 (w), 1693 (s), 1453 (m), 1428 (m), 1376 (m), 1299 (m), 1188 (m), 1161 (m), 1093 (m), 1045 (s), $1019(\mathrm{~m}), 1008(\mathrm{~m}), 991(\mathrm{~m}), 759 \mathrm{~cm}^{-1}(\mathrm{~s})$; accurate mass measurement: $m / z$ calcd. for $\mathrm{C}_{25} \mathrm{H}_{24} \mathrm{INO}_{4}$ $+\mathrm{Na}^{+}$: 552.0642; found: 552.0630; elemental analysis calcd. (\%) for $\mathrm{C}_{25} \mathrm{H}_{24} \mathrm{INO}_{4}: \mathrm{C} 56.72, \mathrm{H} 4.57, \mathrm{~N}$ 2.65, I 23.97; found: C 56.98, H 4.56, N 2.53, I 23.39 .

$\left(3 a R^{*}, 9 a R^{*}, 17 S^{*}\right)-17-\left\{\left[\left(1 R^{*}\right)-1-(\right.\right.$ Methoxycarbonyl)-2-oxocyclopentyl]methyl\}-4-(methoxymethoxy)-2-methyl4,9-dihydro-4,9[1',2']benzeno-3a,9a-propano-1H-benz[f]isoindole-1,3(2H)-dione 24 and mixture of 24 and its C1' epimer 25. A solution of methyl 2-oxocyclopentanecarboxylate $(0.47 \mathrm{~mL}, 95 \%$ content, $500 \mathrm{mg}$, $3.5 \mathrm{mmol})$ was added dropwise to a cold $\left(0^{\circ} \mathrm{C}\right.$, ice-water bath), magnetically-stirred suspension of $\mathrm{NaH}(117 \mathrm{mg}, 60 \%$ content, $2.9 \mathrm{mmol})$ in anhydrous DMF $(5 \mathrm{~mL})$ and the mixture was stirred at this temperature for $1 \mathrm{~h}$. Then, a solution of iodide $23(1.03 \mathrm{~g}, 1.95 \mathrm{mmol})$ was added at once, and the reaction mixture was stirred at $0{ }^{\circ} \mathrm{C}$ for $2 \mathrm{~h}$ and then it was heated at $80{ }^{\circ} \mathrm{C}$ for $63 \mathrm{~h}$. A saturated aqueous solution of $\mathrm{NH}_{4} \mathrm{Cl}(6 \mathrm{~mL})$ and water $(6 \mathrm{~mL})$ were added, and the mixture was extracted with EtOAc $(4 \times 100 \mathrm{~mL})$. The combined organic extracts were dried (anhydrous $\left.\mathrm{Na}_{2} \mathrm{SO}_{4}\right)$ and concentrated in vacuo. The obtained residue (1.3 g) was subjected to column chromatography (100 g 50-200 $\mu \mathrm{m}$ neutral $\mathrm{Al}_{2} \mathrm{O}_{3}$, hexane/EtOAc mixtures). Alkene 18 (135 mg, 17\% yield) was isolated on elution with hexane/EtOAc 4:1. A stereoisomeric mixture of 24 and 25 (about 1:1 mixture of $C 1^{\prime}$-epimers, $628 \mathrm{mg}$, $59 \%$ yield) was obtained as white solid on elution with hexane/EtOAc from 7:3 to 3:2. Crystallization of part of the above product $(100 \mathrm{mg})$ from EtOAc $(0.2 \mathrm{~mL}) /$ hexane $(0.2 \mathrm{~mL})$ gave a mixture of 24 and 25 in a ratio $24 / 25=7: 1\left({ }^{1} \mathrm{H}-\mathrm{NMR}, 22 \mathrm{mg}\right)$. The mother liquors were concentrated in vacuo and crystallized from EtOAc $(0.4 \mathrm{~mL}) /$ hexane $(1.4 \mathrm{~mL})$ gave a mixture of 24 and 25 in a ratio $24 / 25=7.5: 1$ $\left({ }^{1} \mathrm{H}-\mathrm{NMR}, 15 \mathrm{mg}\right)$. Recrystallization of the above product $(37 \mathrm{mg})$ from EtOAc $(0.4 \mathrm{~mL}) /$ hexane $(2 \mathrm{~mL})$ gave two crops (16 and $13.5 \mathrm{mg}$ ) of pure 24 as white solid. M.p. $159-162{ }^{\circ} \mathrm{C}$ (EtOAc/hexane); ${ }^{1} \mathrm{H}-\mathrm{NMR}$ $\left(400 \mathrm{MHz}, \mathrm{CDCl}_{3}\right): \delta=0.80\left(\mathrm{t}^{2} \mathrm{~J}_{(\mathrm{H}, \mathrm{H})}={ }^{3} \mathrm{~J}_{(\mathrm{H}, \mathrm{H})}=12.8 \mathrm{~Hz}, 1 \mathrm{H}, 16-\mathrm{H}_{\mathrm{n}}\right), 1.01\left(\mathrm{t},{ }^{2} \mathrm{~J}_{(\mathrm{H}, \mathrm{H})}={ }^{3} \mathrm{~J}_{(\mathrm{H}, \mathrm{H})}=12.8 \mathrm{~Hz}\right.$, $\left.18-\mathrm{H}_{\mathrm{n}}\right), 1.16\left(\mathrm{dd},{ }^{2} \mathrm{~J}_{(\mathrm{H}, \mathrm{H})}=14.4 \mathrm{~Hz},{ }^{3} \mathrm{~J}_{(\mathrm{H}, \mathrm{H})}=6.4 \mathrm{~Hz}, 1 \mathrm{H}, \mathrm{C} 1-\mathrm{CH}_{\mathrm{a}}\right), 1.57-1.71$ (complex signal, $2 \mathrm{H}, 5^{\prime}-\mathrm{H}_{\mathrm{a}}$ and 17-H), $1.78\left(\mathrm{dd}^{2} \mathrm{~J}_{(\mathrm{H}, \mathrm{H})}=14.0 \mathrm{~Hz},{ }^{3} \mathrm{~J}_{(\mathrm{H}, \mathrm{H})}=6.8 \mathrm{~Hz}, 1 \mathrm{H}, \mathrm{C} 1-\mathrm{CH}_{\mathrm{b}}\right), 1.81-1.97$ (complex signal, $4^{\prime}-\mathrm{H}_{\mathrm{a}}$ and $\left.4^{\prime}-\mathrm{H}_{\mathrm{b}}\right), 2.07\left(\mathrm{ddd},{ }^{2} \mathrm{~J}_{(\mathrm{H}, \mathrm{H})}=12.8 \mathrm{~Hz},{ }^{3} \mathrm{~J}_{(\mathrm{H}, \mathrm{H})}=6.0 \mathrm{~Hz},{ }^{4} \mathrm{~J}_{(\mathrm{H}, \mathrm{H})}=1.2 \mathrm{~Hz}, 16-\mathrm{H}_{\mathrm{x}}\right), 2.13-2.21$ (overlapped $\left.\mathrm{dd},{ }^{2} \mathrm{~J}_{(\mathrm{H}, \mathrm{H})}=19.2 \mathrm{~Hz},{ }^{3} \mathrm{~J}_{(\mathrm{H}, \mathrm{H})}=10.0 \mathrm{~Hz}, 3^{\prime}-\mathrm{H}_{\mathrm{a}}\right), 2.18-2.23$ (overlapped dd, ${ }^{2} \mathrm{~J}_{(\mathrm{H}, \mathrm{H})}=12.8 \mathrm{~Hz},{ }^{3} \mathrm{~J}_{(\mathrm{H}, \mathrm{H})}=$ $\left.6.0 \mathrm{~Hz},{ }^{4} \mathrm{~J}(\mathrm{H}, \mathrm{H})=1.6 \mathrm{~Hz}, 18-\mathrm{H}_{\mathrm{x}}\right), 2.33\left(\mathrm{dddd},{ }^{2} \mathrm{~J}_{(\mathrm{H}, \mathrm{H})}=19.2 \mathrm{~Hz},{ }^{3} \mathrm{~J}_{(\mathrm{H}, \mathrm{H})}=8.6 \mathrm{~Hz},{ }^{3} \mathrm{~J}_{(\mathrm{H}, \mathrm{H})}=4.8 \mathrm{~Hz},{ }^{4} \mathrm{~J}_{(\mathrm{H}, \mathrm{H})}\right.$ $\left.=1.2 \mathrm{~Hz}, 1 \mathrm{H}, 3^{\prime}-\mathrm{H}_{\mathrm{b}}\right), 2.42-2.52\left(\mathrm{~m}, 1 \mathrm{H}, 5^{\prime}-\mathrm{H}_{\mathrm{b}}\right), 2.45\left(\mathrm{~s}, 3 \mathrm{H}, \mathrm{N}-\mathrm{CH}_{3}\right), 3.62\left(3 \mathrm{H}, \mathrm{COOCH}_{3}\right), 3.77(\mathrm{~s}, 3 \mathrm{H}$, $\left.\mathrm{OCH}_{3}\right), 4.35(\mathrm{~s}, 1 \mathrm{H}, 9-\mathrm{H}), 5.35\left(\mathrm{~s}, 2 \mathrm{H}, \mathrm{OCH}_{2} \mathrm{O}\right), 7.10\left(\mathrm{dt},{ }^{4} \mathrm{~J}_{(\mathrm{H}, \mathrm{H})}=1.6 \mathrm{~Hz},{ }^{3} \mathrm{~J}_{(\mathrm{H}, \mathrm{H})}=7.4 \mathrm{~Hz}, 1 \mathrm{H}, 7-\mathrm{H}\right)$, $7.15\left(\mathrm{dt},{ }^{4} \mathrm{~J}_{(\mathrm{H}, \mathrm{H})}=1.6 \mathrm{~Hz},{ }^{3} \mathrm{~J}_{(\mathrm{H}, \mathrm{H})}=7.4 \mathrm{~Hz}, 6-\mathrm{H}\right), 7.18-7.20(\mathrm{~m}, 8-\mathrm{H}), 7.24\left(\mathrm{dt},{ }^{4} \mathrm{~J}_{(\mathrm{H}, \mathrm{H})}=1.6 \mathrm{~Hz},{ }^{3} \mathrm{~J}_{(\mathrm{H}, \mathrm{H})}=\right.$ $7.2 \mathrm{~Hz}, 12-\mathrm{H}), 7.29\left(\mathrm{dt}^{4} \mathrm{~J}_{(\mathrm{H}, \mathrm{H})}=1.6 \mathrm{~Hz},{ }^{3} \mathrm{~J}_{(\mathrm{H}, \mathrm{H})}=7.2 \mathrm{~Hz}, 13-\mathrm{H}\right), 7.33-7.36(\mathrm{~m}, 1 \mathrm{H}, 11-\mathrm{H}), 7.70-7.72(\mathrm{~m}$, 1H, 14-H), 7.72-7.74 ppm (m, 1H, 5-H); ${ }^{13} \mathrm{C}-\mathrm{NMR}\left(100.6 \mathrm{MHz}, \mathrm{CDCl}_{3}\right): \delta=19.4\left(\mathrm{CH}_{2}, \mathrm{C}^{\prime}\right), 24.7\left(\mathrm{CH}_{3}\right.$, $\left.\mathrm{N}-\mathrm{CH}_{3}\right), 32.6\left(\mathrm{CH}_{2}, \mathrm{C}^{\prime}\right), 36.7(\mathrm{CH}, \mathrm{C} 17), 37.3\left(\mathrm{CH}_{2}, \mathrm{Cl}^{\prime}\right), 38.2\left(\mathrm{CH}_{2}, \mathrm{C} 1-\mathrm{CH}_{2}\right), 38.4\left(\mathrm{CH}_{2}, \mathrm{C} 16\right), 38.5$ $\left(\mathrm{CH}_{2}, \mathrm{C} 18\right), 48.6(\mathrm{CH}, \mathrm{C} 9), 52.6\left(\mathrm{CH}_{3}, \mathrm{COOCH}_{3}\right), 56.3\left(\mathrm{CH}_{3}, \mathrm{OCH}_{3}\right), 60.1(\mathrm{C}, \mathrm{C} 1), 64.8(\mathrm{C}, \mathrm{C} 9 \mathrm{a}), 65.3(\mathrm{C}$, C3a), $87.1(\mathrm{C}, \mathrm{C} 4), 94.1\left(\mathrm{CH}_{2}, \mathrm{OCH}_{2} \mathrm{O}\right), 122.7(\mathrm{CH}, \mathrm{C} 5), 124.3(\mathrm{CH}, \mathrm{C} 14), 124.7(\mathrm{CH}, \mathrm{C} 8), 126.3(\mathrm{CH}$, C11), 126.9 (2 CH, C6 and C13), 127.1 (CH, C7), 127.2 (CH, C12), 138.2 (C, C8a), 138.3 (C, C10), 139.5 (C, 
C4a), 139.9 (C, C15), $170.5\left(\mathrm{C}, \mathrm{COOCH}_{3}\right), 177.5$ (C, C3), 179.6 (C, C1), 214.0 ppm (C, C2'); IR (ATR): $\check{\text { v }}$ 2945 (w), 1767 (w), 1746 (w), $1713(\mathrm{~m}), 1695$ (s), $1452(\mathrm{~m}), 1439(\mathrm{~m}), 1429$ (m), 1377 (m), 1305 (m), 1256 (m), $1238(\mathrm{~m}), 1204(\mathrm{~m}), 1186(\mathrm{~m}), 1165(\mathrm{~m}), 1046(\mathrm{~s}), 10113(\mathrm{~m}), 997(\mathrm{~m}), 752 \mathrm{~cm}^{-1}(\mathrm{~m})$; accurate mass measurement: $\mathrm{m} / \mathrm{z}$ calcd. for $\mathrm{C}_{32} \mathrm{H}_{33} \mathrm{NO}_{7}+\mathrm{H}^{+}$: 544.2330; found: 544.2329; elemental analysis calcd. (\%) for $\mathrm{C}_{32} \mathrm{H}_{33} \mathrm{NO}_{7}$ : C 70.70, H 6.12, N 2.58; found: C 70.38, H 5.95, N 2.39.

Significant ${ }^{1} \mathrm{H}-\mathrm{NMR}$ data of $\mathbf{2 5}$ from the 1:1 mixture $\mathbf{2 4}$ and $\mathbf{2 5}$ and analytical data from this mixture: $\left.{ }^{1} \mathrm{H}-\mathrm{NMR}\left(400 \mathrm{MHz}, \mathrm{CDCl}_{3}\right): \delta=0.79\left(\mathrm{t},{ }^{2} J_{(\mathrm{H}, \mathrm{H})}={ }^{3} J_{(\mathrm{H}, \mathrm{H}}\right)=12.8 \mathrm{~Hz}, 1 \mathrm{H}, 16-\mathrm{H}_{\mathrm{n}}\right), 1.03\left(\mathrm{t},{ }^{2} J_{(\mathrm{H}, \mathrm{H})}={ }^{3} J_{(\mathrm{H}, \mathrm{H})}=\right.$ $\left.12.8 \mathrm{~Hz}, 18-\mathrm{H}_{\mathrm{n}}\right), 5.34\left(\mathrm{~d},{ }^{2} \mathrm{~J}_{(\mathrm{H}, \mathrm{H})}=5.2 \mathrm{~Hz}, 1 \mathrm{H}, \mathrm{OCH}_{\mathrm{a}} \mathrm{O}\right), 5.37\left(\mathrm{~d},{ }^{2} \mathrm{~J}_{(\mathrm{H}, \mathrm{H})}=5.2 \mathrm{~Hz}, 1 \mathrm{H}, \mathrm{OCH}_{\mathrm{b}} \mathrm{O}\right)$; accurate mass measurement: $m / z$ calcd. for $\mathrm{C}_{32} \mathrm{H}_{33} \mathrm{NO}_{7}+\mathrm{H}^{+}$: 544.2330; found: 544.2346; elemental analysis calcd. (\%) for $\mathrm{C}_{32} \mathrm{H}_{33} \mathrm{NO}_{7}$ : C 70.70, H 6.12, N 2.58; found: C 70.45, H 6.13, N 2.46.

$\left(3 a R^{*}, 9 a R^{*}, 17 S^{*}\right)-17-\left\{\left[\left(1^{\prime} R^{*}\right)\right.\right.$-1-(Methoxycarbonyl)-2-oxocyclopent-3-en-1-yl]methyl\}-4-(methoxymethoxy)-2methyl-4,9-dihydro-4,9[1' $\left.2^{\prime}\right]$ benzeno-3a,9a-propano-1H-benz[f]isoindole-1,3(2H)-dione (26). Trimethylsilyl trifluoromethanesulfonate $(80 \mu \mathrm{L}, 98 \%$ content, $96 \mathrm{mg}, 0.43 \mathrm{mmol})$ was added at once to a cold $\left(0{ }^{\circ} \mathrm{C}\right.$, ice-water bath) solution of keto ester $24(101 \mathrm{mg}, 0.19 \mathrm{mmol})$ and anhydrous $\mathrm{Et}_{3} \mathrm{~N}(0.13 \mathrm{~mL}, 94 \mathrm{mg}$, $0.93 \mathrm{mmol})$ in anhydrous $\mathrm{CH}_{2} \mathrm{Cl}_{2}(1 \mathrm{~mL})$, under an Ar atmosphere, and the mixture was stirred at room temperature for $30 \mathrm{~min}$. The solution was cooled to $0{ }^{\circ} \mathrm{C}$ (ice-water bath), a saturated aqueous solution of $\mathrm{NaHCO}_{3}(1 \mathrm{~mL})$ was added, the organic phase was separated, and the aqueous one was extracted with $\mathrm{CH}_{2} \mathrm{Cl}_{2}(2 \times 4 \mathrm{~mL})$. The combined organic phases were dried (anhydrous $\left.\mathrm{Na}_{2} \mathrm{SO}_{4}\right)$ and concentrated in vacuo to give a brown oily residue, of the corresponding trimethylsilyl enol ether (118 mg), which was used as such in the next step. $\mathrm{Pd}(\mathrm{OAc})_{2}$ ( $43.5 \mathrm{mg}, 98 \%$ content, $0.19 \mathrm{mmol}$ ) was added to a solution of the above enol ether $(118 \mathrm{mg})$ in anhydrous DMSO $(5 \mathrm{~mL})$ and the mixture was stirred for $16 \mathrm{~h}$ at room temperature. The suspension was filtered through a pad of Celite ${ }^{\circledR}$, and the solid was washed with EtOAc $(10 \mathrm{~mL})$. The combined filtrate and washings were concentrated in vacuo, the residue was taken in EtOAc $(15 \mathrm{~mL})$ and was washed with water $(15 \mathrm{~mL})$. The aqueous phase was extracted with EtOAc $(2 \times 20 \mathrm{~mL})$. The combined organic phase and extracts were washed with brine $\left(2 \times 25 \mathrm{~mL}\right.$ ), dried (anhydrous $\left.\mathrm{Na}_{2} \mathrm{SO}_{4}\right)$ and concentrated in vacuo. The brown oily residue (120 mg) was subjected to column chromatography (10 g, 50-200 $\mu \mathrm{m}$ basic $\mathrm{Al}_{2} \mathrm{O}_{3}$, hexane/EtOAc mixtures). On elution with hexane/EtOAc from 9:1 to 3:2, enone $26(69 \mathrm{mg}$, 68\% yield from 24) was obtained as white solid. m.p. $160-162{ }^{\circ} \mathrm{C}($ EtOAc/hexane $) ;{ }^{1} \mathrm{H}-\mathrm{NMR}\left(400 \mathrm{MHz}, \mathrm{CDCl}_{3}\right): \delta=0.86(\mathrm{t}$, $\left.{ }^{2} J_{(\mathrm{H}, \mathrm{H})}={ }^{3} J_{(\mathrm{H}, \mathrm{H})}=12.8 \mathrm{~Hz}, 1 \mathrm{H}, 16-\mathrm{H}_{\mathrm{n}}\right), 1.04\left(\mathrm{t},{ }^{2} J_{(\mathrm{H}, \mathrm{H})}={ }^{3} J_{(\mathrm{H}, \mathrm{H})}=12.8 \mathrm{~Hz}, 1 \mathrm{H}, 18-\mathrm{H}_{\mathrm{n}}\right), 1.33\left(\mathrm{dd},{ }^{2} J_{(\mathrm{H}, \mathrm{H})}=\right.$ $\left.14.4 \mathrm{~Hz},{ }^{3} J_{(\mathrm{H}, \mathrm{H})}=6.4 \mathrm{~Hz}, 1 \mathrm{H}, \mathrm{Cl}^{\prime}-\mathrm{CH}_{\mathrm{a}}\right), 1.55-1.68(\mathrm{~m}, 1 \mathrm{H}, 17-\mathrm{H}), 1.84\left(\mathrm{dd},{ }^{2} J_{(\mathrm{H}, \mathrm{H})}=14.4 \mathrm{~Hz},{ }^{3} J_{(\mathrm{H}, \mathrm{H})}=\right.$ $\left.6.4 \mathrm{~Hz}, 1 \mathrm{H}, \mathrm{Cl}^{\prime}-\mathrm{CH}_{\mathrm{b}}\right), 2.07\left(\mathrm{ddd}^{2} J_{(\mathrm{H}, \mathrm{H})}=12.8 \mathrm{~Hz},{ }^{3} J_{(\mathrm{H}, \mathrm{H})}=5.8 \mathrm{~Hz},{ }^{4} J_{(\mathrm{H}, \mathrm{H})}=1.6 \mathrm{~Hz}, 1 \mathrm{H}, 16-\mathrm{H}_{\mathrm{x}}\right), 2.19$ $\left(\mathrm{ddd},{ }^{2} J_{(\mathrm{H}, \mathrm{H})}=13.2 \mathrm{~Hz},{ }^{3} J_{(\mathrm{H}, \mathrm{H})}=6.0 \mathrm{~Hz},{ }^{4} J_{(\mathrm{H}, \mathrm{H})}=1.3 \mathrm{~Hz}, 1 \mathrm{H}, 18-\mathrm{H}_{\mathrm{x}}\right), 2.42$ (overlapped dt, ${ }^{2} J_{(\mathrm{H}, \mathrm{H})}=$ $\left.19.2 \mathrm{~Hz},{ }^{3} J_{(\mathrm{H}, \mathrm{H})}=2.4 \mathrm{~Hz}, 1 \mathrm{H}, 5^{\prime}-\mathrm{H}_{\mathrm{a}}\right), 2.44$ (overlapped s, $\left.3 \mathrm{H}, \mathrm{N}-\mathrm{CH}_{3}\right), 3.22\left(\mathrm{dt}^{2} J_{(\mathrm{H}, \mathrm{H})}=19.2 \mathrm{~Hz},{ }^{3} J_{(\mathrm{H}, \mathrm{H})}\right.$ $\left.=2.6 \mathrm{~Hz}, 1 \mathrm{H}, 5^{\prime}-\mathrm{H}_{\mathrm{b}}\right), 3.62\left(\mathrm{~s}, 3 \mathrm{H}, \mathrm{COOCH}_{3}\right), 3.77\left(\mathrm{~s}, 3 \mathrm{H}, \mathrm{OCH}_{3}\right), 4.35(\mathrm{~s}, 1 \mathrm{H}, 9-\mathrm{H}), 5.34\left(\mathrm{~s}, 2 \mathrm{H}, \mathrm{OCH}_{2} \mathrm{O}\right)$, $6.09\left(\mathrm{dt}^{3}{ }^{3} J_{(\mathrm{H}, \mathrm{H})}=5.6 \mathrm{~Hz},{ }^{3} J_{(\mathrm{H}, \mathrm{H})}=2.0 \mathrm{~Hz}, 1 \mathrm{H}, 3^{\prime}-\mathrm{H}\right), 7.11\left(\mathrm{dt}^{3} J_{(\mathrm{H}, \mathrm{H})}=7.2 \mathrm{~Hz},{ }^{4} J_{(\mathrm{H}, \mathrm{H})}=1.6 \mathrm{~Hz}, 1 \mathrm{H}, 7-\mathrm{H}\right)$, $7.15\left(\mathrm{dt}^{3}{ }^{3} J_{(\mathrm{H}, \mathrm{H})}=7.2 \mathrm{~Hz},{ }^{4} J_{(\mathrm{H}, \mathrm{H})}=1.6 \mathrm{~Hz}, 1 \mathrm{H}, 6-\mathrm{H}\right), 7.19\left(\mathrm{ddm},{ }^{3} J_{(\mathrm{H}, \mathrm{H})}=6.8 \mathrm{~Hz},{ }^{4} J_{(\mathrm{H}, \mathrm{H})}=1.6 \mathrm{~Hz}, 1 \mathrm{H}\right.$, $8-\mathrm{H}), 7.25\left(\mathrm{dt},{ }^{3} J_{(\mathrm{H}, \mathrm{H})}=7.2 \mathrm{~Hz},{ }^{4} J_{(\mathrm{H}, \mathrm{H})}=1.6 \mathrm{~Hz}, 1 \mathrm{H}, 12-\mathrm{H}\right), 7.28\left(\mathrm{dt},{ }^{3} J_{(\mathrm{H}, \mathrm{H})}=7.2 \mathrm{~Hz},{ }^{4} J_{(\mathrm{H}, \mathrm{H})}=1.6 \mathrm{~Hz}\right.$, $1 \mathrm{H}, 13-\mathrm{H}), 7.35\left(\mathrm{ddm},{ }^{3} J_{(\mathrm{H}, \mathrm{H})}=6.4 \mathrm{~Hz},{ }^{4} J_{(\mathrm{H}, \mathrm{H})}=1.6 \mathrm{~Hz}, 1 \mathrm{H}, 11-\mathrm{H}\right) 7.69-7.74 \mathrm{ppm}$ (complex signal, $3 \mathrm{H}$, $4^{\prime}-\mathrm{H}, 5-\mathrm{H}$ and $\left.14-\mathrm{H}\right) ;{ }^{13} \mathrm{C}-\mathrm{NMR}\left(100.6 \mathrm{MHz}, \mathrm{CDCl}_{3}\right): \delta=24.7\left(\mathrm{CH}_{3}, \mathrm{~N}-\mathrm{CH}_{3}\right), 36.8(\mathrm{CH}, \mathrm{C} 17), 38.3\left(\mathrm{CH}_{2}\right.$, $\left.\mathrm{Cl}^{\prime}-\mathrm{CH}_{2}\right), 38.6\left(\mathrm{CH}_{2}, \mathrm{C} 16\right), 38.7\left(\mathrm{CH}_{2}, \mathrm{C} 18\right), 38.9\left(\mathrm{CH}_{2}, \mathrm{C}^{\prime}\right), 48.6(\mathrm{CH}, \mathrm{C} 9), 52.8\left(\mathrm{CH}_{3}, \mathrm{COOCH}_{3}\right), 56.4$ $\left(\mathrm{CH}_{3}, \mathrm{OCH}_{3}\right), 57.6\left(\mathrm{C}, \mathrm{Cl}^{\prime}\right), 64.7(\mathrm{C}, \mathrm{C} 9 \mathrm{a}), 65.3(\mathrm{C}, \mathrm{C} 3 \mathrm{a}), 87.2(\mathrm{C}, \mathrm{C} 4), 94.1\left(\mathrm{OCH}_{2} \mathrm{O}\right), 122.7(\mathrm{CH}, \mathrm{C} 5)$, $124.3(\mathrm{CH}, \mathrm{C} 14), 124.7(\mathrm{CH}, \mathrm{C} 8), 126.3(\mathrm{CH}, \mathrm{C} 11), 126.89(\mathrm{CH})$ and $126.91(\mathrm{CH})(\mathrm{C} 6$ and $\mathrm{C} 13), 127.1(\mathrm{CH}$, C7), $127.2(\mathrm{CH}, \mathrm{C} 12), 131.6\left(\mathrm{CH}, \mathrm{C}^{\prime}\right), 138.20(\mathrm{C})$ and 138.23 (C) (C8a and $\left.\mathrm{C} 10\right), 139.5$ (C, C4a), 139.8 (C, C15), $163.9\left(\mathrm{CH}, \mathrm{C4}^{\prime}\right), 170.3\left(\mathrm{COOCH}_{3}\right), 177.4(\mathrm{C}, \mathrm{C} 3), 179.6(\mathrm{C}, \mathrm{C} 1), 204.8 \mathrm{ppm}\left(\mathrm{C}, \mathrm{C2} 2^{\prime}\right)$; IR (ATR): $\check{v}=$ $2940(\mathrm{w}), 1767(\mathrm{w}), 1741(\mathrm{~m}), 1695(\mathrm{~s}), 1454(\mathrm{~m}), 1423(\mathrm{~m}), 1240(\mathrm{~m}), 1186(\mathrm{~m}), 1168(\mathrm{~m}), 1054(\mathrm{~m}), 1031$ (m), $1013(\mathrm{~m}), 984(\mathrm{~s}), 760(\mathrm{~s}), 749 \mathrm{~cm}^{-1}(\mathrm{~m})$; accurate mass measurement: $m / z$ calcd. for $\mathrm{C}_{32} \mathrm{H}_{31} \mathrm{NO}_{7}+$ 
$\mathrm{Na}^{+}$: 564.1993; found: 564.1993; elemental analysis calcd. (\%) for $\mathrm{C}_{32} \mathrm{H}_{31} \mathrm{NO}_{7} \cdot 0.25 \mathrm{H}_{2} \mathrm{O}: \mathrm{C} 70.38, \mathrm{H} 5.81$, N 2.56; found: C 70.28, H 5.73, N 2.39.

Stereoisomeric mixture of $\mathbf{2 6}$ and its $C 1^{\prime}$ epimer (27). Trimethylsilyl trifluoromethanesulfonate (310 $\mu \mathrm{L}$, $98 \%$ content, $372 \mathrm{mg}, 1.68 \mathrm{mmol})$ was added at once to a cold $\left(0{ }^{\circ} \mathrm{C}\right.$, ice-water bath) solution of a stereoisomeric mixture of keto esters 24 and 25 (approximate ratio $24 / 25=1: 1,400 \mathrm{mg}, 0.74 \mathrm{mmol}$ ) and anhydrous $\mathrm{Et}_{3} \mathrm{~N}(0.51 \mathrm{~mL}, 370 \mathrm{mg}, 3.7 \mathrm{mmol})$ in anhydrous $\mathrm{CH}_{2} \mathrm{Cl}_{2}(3 \mathrm{~mL})$ under an $\mathrm{Ar}$ atmosphere, and the mixture was stirred at room temperature for $30 \mathrm{~min}$. The solution was cooled to $0{ }^{\circ} \mathrm{C}$ (ice-water bath), a saturated aqueous solution of $\mathrm{NaHCO}_{3}(3 \mathrm{~mL})$ was added, the organic phase was separated and the aqueous one was extracted with $\mathrm{CH}_{2} \mathrm{Cl}_{2}(2 \times 6 \mathrm{~mL})$. The combined organic phases were dried (anhydrous $\mathrm{Na}_{2} \mathrm{SO}_{4}$ ) and concentrated in vacuo to give a brown oily residue, mixture of the corresponding trimethylsilyl enol ethers $(412 \mathrm{mg})$, which was used as such in the next step. Accurate mass measurement: $m / z$ calcd. for $\mathrm{C}_{35} \mathrm{H}_{41} \mathrm{NO}_{7} \mathrm{Si}+\mathrm{H}^{+}$: 616.2725; found: 616.2731.

$\mathrm{Pd}(\mathrm{OAc})_{2}$ (169 $\mathrm{mg}, 98 \%$ content, $0.74 \mathrm{mmol}$ ) was added to a solution of the above stereoisomeric mixture of enol ethers $(412 \mathrm{mg})$ in anhydrous DMSO $(15 \mathrm{~mL})$ and the mixture was stirred at room temperature for $16 \mathrm{~h}$. The suspension was filtered through a pad of Celite ${ }^{\circledR}$, and the solid was washed with EtOAc $(20 \mathrm{~mL})$. The combined filtrate and washings were concentrated in vacuo, the residue was taken in EtOAc $(20 \mathrm{~mL})$ and was washed with water $(20 \mathrm{~mL})$. The aqueous phase was extracted with EtOAc $(2 \times 20 \mathrm{~mL})$. The combined organic phase and extracts were washed with brine $(2 \times 25 \mathrm{~mL})$, dried (anhydrous $\mathrm{Na}_{2} \mathrm{SO}_{4}$ ) and concentrated in vacuo to give a brown oily residue ( $390 \mathrm{mg}$ ), which was subjected to column chromatography ( $10 \mathrm{~g}, 50-200 \mu \mathrm{m}$ basic $\mathrm{Al}_{2} \mathrm{O}_{3}$, hexane/EtOAc mixtures). On elution with hexane/EtOAc 3:2, a stereoisomeric mixture of enones $\mathbf{2 6}$ and $\mathbf{2 7}$ in an approximate ratio $\mathbf{2 6} / \mathbf{2 7}=1: 1(301 \mathrm{mg}, 75 \%$ yield from $24+25)$ was obtained as white solid. m.p. $76-86{ }^{\circ} \mathrm{C}$ (EtOAc/hexane); accurate mass measurement: $m / z$ calcd. for $\mathrm{C}_{32} \mathrm{H}_{31} \mathrm{NO}_{7}+\mathrm{H}^{+}$: 542.2173; found: 542.2179; elemental analysis calcd. (\%) for $\mathrm{C}_{32} \mathrm{H}_{31} \mathrm{NO}_{7} 1.5 \mathrm{H}_{2} \mathrm{O}: \mathrm{C} 67.59, \mathrm{H} 6.03, \mathrm{~N} 2.46$; found: $\mathrm{C} 67.88$, H 5.78, N 2.29.

Methyl 2-methyl-1,3-dioxo-2,3,5,6-tetrahydro-1H,4H-3a,7,8-(epiprop[2]ene[1,1,3]triyl)-5,8a-methanocyclo hepta[c]pyrrole-7(8H)-carboxylate 13.

(a) $\mathrm{NaBH}_{4}(67 \mathrm{mg}, 1.72 \mathrm{mmol})$ was added portionwise to a cold $\left(-40^{\circ} \mathrm{C}\right)$ solution of a diastereomeric mixture of enones 26 and $27(233 \mathrm{mg}, 0.43 \mathrm{mmol})$ and $\mathrm{CeCl}_{3} \cdot 7 \mathrm{H}_{2} \mathrm{O}(417 \mathrm{mg}, 1.12 \mathrm{mmol})$ in a mixture of THF $(4.5 \mathrm{~mL})$ and $\mathrm{MeOH}(5 \mathrm{~mL})$ and the mixture was stirred at this temperature for $1 \mathrm{~h}$. A saturated aqueous solution of $\mathrm{NaHCO}_{3}(2 \mathrm{~mL})$ and water $(2 \mathrm{~mL})$ were added and the mixture was extracted with $\mathrm{CH}_{2} \mathrm{Cl}_{2}(3 \times 15 \mathrm{~mL})$. The combined organic phases were dried (anhydrous $\mathrm{Na}_{2} \mathrm{SO}_{4}$ ) and concentrated in vacuo to give a complex diastereoisomeric mixture of cyclopentenols $28(172 \mathrm{mg})$ as white solid, that was used as such in the next step.

(b) $\quad p$ - $\mathrm{TsOH} \mathrm{H} \mathrm{H}_{2} \mathrm{O}(16.4 \mathrm{mg}, 0.09 \mathrm{mmol})$ was added to a solution of the above cyclopentenols 28 $(172 \mathrm{mg})$ in benzene $(15 \mathrm{~mL})$ and the solution was heated under reflux for $14 \mathrm{~h}$ with azeotropic elimination of water with a Dean-Stark equipment. Then, the solution was allowed to cool to room temperature and was treated with solid $\mathrm{K}_{2} \mathrm{CO}_{3}$ (about $100 \mathrm{mg}$ ). The suspension was filtered and the filtrate was concentrated in vacuo to give cyclopentadiene alcohol 29 as light brown solid $(180 \mathrm{mg})$ that was used as such in the next step.

(c) A suspension of $30 \% \mathrm{KH}$ in mineral oil ( $150 \mathrm{mg}$, about $1.1 \mathrm{mmol}$ ) was placed in a three-necked flask, provided with a low temperature thermometer and an Ar atmosphere. The mineral oil was removed by washing with anhydrous THF $(5 \times 5 \mathrm{~mL})$ and after the last washing anhydrous THF $(12 \mathrm{~mL})$ was added. The mixture was cooled to $0{ }^{\circ} \mathrm{C}$ with an ice-water bath and a solution of the above product $29(180 \mathrm{mg})$ in anhydrous THF $(12 \mathrm{~mL})$ was added dropwise with magnetic stirring and the mixture was stirred at room temperature for $1 \mathrm{~h}$. The excess $\mathrm{KH}$ was destroyed by careful addition of $2 \mathrm{~N} \mathrm{HCl}(1 \mathrm{~mL})$ plus $5 \mathrm{~N} \mathrm{HCl}(0.5 \mathrm{~mL})$ under an $\mathrm{Ar}$ atmosphere. The solution 
was dried (anhydrous $\mathrm{MgSO}_{4}$, about $3 \mathrm{~g}$ ), filtered under vacuum and concentrated under reduced pressure to give an oily residue $(263 \mathrm{mg})$ that was subjected to column chromatography ( $20 \mathrm{~g}$, 35-70 $\mu \mathrm{m}$ silica gel, hexane/EtOAc mixtures). Compound 13 (37 mg, 30\% yield from $26+27$ ) was obtained as a light yellow solid, on elution with hexane/EtOAc 4:1. The ${ }^{1} \mathrm{H}-\mathrm{NMR}$ data of this compound coincide with those previously described.

7a-(Methoxycarbonyl)-4-(methylcarbamoyl)-3a,4,5,6,7,7a-hexahydro-1H-1,4,6-(epiethane[1,1,2]triyl)indene-9carboxylic acid (30). $\mathrm{KOH} \mathrm{(236} \mathrm{mg,} 4.2 \mathrm{mmol})$ was added to a solution of compound 13 (121 mg, $0.42 \mathrm{mmol})$ in a mixture of THF $(2 \mathrm{~mL})$ and $\mathrm{MeOH}(2 \mathrm{~mL})$ and the mixture was stirred at room temperature for $15 \mathrm{~h}$. Water $(8 \mathrm{~mL})$ was added and the mixture was extracted with EtOAc $(3 \times 10 \mathrm{~mL})$. The aqueous phase was made acidic with $1 \mathrm{~N} \mathrm{HCl}(6 \mathrm{~mL})$ and it was extracted with EtOAc $(3 \times 10 \mathrm{~mL})$. The combined organic phases were washed with water $\left(2 \times 10 \mathrm{~mL}\right.$ ), dried (anhydrous $\left.\mathrm{Na}_{2} \mathrm{SO}_{4}\right)$ and concentrated in vacuo to give amide acid (30) (96 mg, 75\% yield) as yellow solid. An analytical sample of (30) (13 mg) was obtained as yellow solid by crystallization of a sample (19 $\mathrm{mg})$ of the above product in $\mathrm{MeOH}(0.5 \mathrm{~mL})$. m.p. $193-194{ }^{\circ} \mathrm{C}(\mathrm{MeOH}) ;{ }^{1} \mathrm{H}-\mathrm{NMR}\left(\mathrm{CD}_{3} \mathrm{OD}\right): \delta=1.81\left(\mathrm{~d},{ }^{3} J_{(\mathrm{H}, \mathrm{H})}=\right.$ $2.8 \mathrm{~Hz}, 2 \mathrm{H}, 7-\mathrm{H}_{\mathrm{a}}$ and $\left.7-\mathrm{H}_{\mathrm{b}}\right), 1.90\left(\mathrm{dd},{ }^{2} J_{(\mathrm{H}, \mathrm{H})}=11.6 \mathrm{~Hz},{ }^{4} J_{(\mathrm{H}, \mathrm{H})}=2.4 \mathrm{~Hz}, 1 \mathrm{H}\right)$ and $1.94\left(\mathrm{dd},{ }^{2} J_{(\mathrm{H}, \mathrm{H})}=\right.$ $\left.11.6 \mathrm{~Hz},{ }^{4} J_{(\mathrm{H}, \mathrm{H})}=2.4 \mathrm{~Hz}, 1 \mathrm{H}\right)\left(5-\mathrm{H}_{\mathrm{anti}}\right.$ and $\left.8-\mathrm{H}_{\mathrm{anti}}\right), 2.23\left[\mathrm{dd},{ }^{2} J_{(\mathrm{H}, \mathrm{H})}=11.6 \mathrm{~Hz},{ }^{3} J_{(\mathrm{H}, \mathrm{H})}=4.0 \mathrm{~Hz}, 1 \mathrm{H}\right.$, $\left.5-\mathrm{H}_{\mathrm{syn}}\right), 2.41\left[\mathrm{dd},{ }^{2} J_{(\mathrm{H}, \mathrm{H})}=11.6 \mathrm{~Hz},{ }^{3} J_{(\mathrm{H}, \mathrm{H})}=4.0 \mathrm{~Hz}, 1 \mathrm{H}, 8-\mathrm{H}_{\mathrm{syn}}\right), 2.46-2.49(\mathrm{~m}, 1 \mathrm{H}, 6-\mathrm{H}), 2.67[\mathrm{~s}, 3 \mathrm{H}$, $\left.\mathrm{N}-\mathrm{CH}_{3}\right], 2.96-2.98[\mathrm{~m}, 2 \mathrm{H}, 1-\mathrm{H}$ and $3 \mathrm{a}-\mathrm{H}], 3.58\left[\mathrm{~s}, 3 \mathrm{H}, \mathrm{OCH}_{3}\right], 6.19\left(\mathrm{ddd},{ }^{3} J_{(\mathrm{H}, \mathrm{H})}=5.6 \mathrm{~Hz},{ }^{3} J_{(\mathrm{H}, \mathrm{H})}=\right.$ $\left.2.8 \mathrm{~Hz},{ }^{4} J_{(\mathrm{H}, \mathrm{H})}=1.2 \mathrm{~Hz}, 1 \mathrm{H}, 2-\mathrm{H}\right), 6.34 \mathrm{ppm}\left(\mathrm{ddd},{ }^{3} J_{(\mathrm{H}, \mathrm{H})}=5.6 \mathrm{~Hz},{ }^{3} J_{(\mathrm{H}, \mathrm{H})}=2.8 \mathrm{~Hz},{ }^{4} J_{(\mathrm{H}, \mathrm{H})}=1.2 \mathrm{~Hz}, 1 \mathrm{H}\right.$, 3-H); ${ }^{13} \mathrm{C}-\mathrm{NMR}\left(\mathrm{CD}_{3} \mathrm{OD}\right): \delta=26.7\left[\mathrm{CH}_{3}, \mathrm{~N}-\mathrm{CH}_{3}\right], 35.7(\mathrm{CH}, \mathrm{C} 6), 36.2\left(\mathrm{CH}_{2}, \mathrm{C} 7\right), 44.3\left(\mathrm{CH}_{2}, \mathrm{C} 5\right), 44.6$ $\left(\mathrm{CH}_{2}, \mathrm{C} 8\right), 52.2\left(\mathrm{CH}_{3}, \mathrm{OCH}_{3}\right), 59.16(\mathrm{CH}, \mathrm{C} 3 \mathrm{a}), 59.20(\mathrm{CH}, \mathrm{C} 1), 63.4(\mathrm{C}, \mathrm{C} 9), 65.3(\mathrm{C}, \mathrm{C} 4), 68.8(\mathrm{C}, \mathrm{C} 7 \mathrm{a})$, $136.0(\mathrm{CH}, \mathrm{C} 3), 138.8(\mathrm{CH}, \mathrm{C} 2), 176.3(\mathrm{C}, \mathrm{CON}), 176.5\left(\mathrm{C}, \mathrm{COOCH}_{3}\right), 177.7(\mathrm{C}, \mathrm{COOH})$; IR (ATR): $\check{v}=$ 3409 (m), 3200-2500 [broad band, max. at $2981(\mathrm{w}), 2949(\mathrm{w}), 2930(\mathrm{w}), 2847(\mathrm{w})$ ], $1731(\mathrm{~s}), 1709(\mathrm{~s})$, 1632 (s), 1531 (s), 1418 (m), 1294 (m), 1280 (m), 1258 (s), 1246 (s), 1209 (m), 1188 (s), 1137 (m), 1105 (m), $1034(\mathrm{~m}), 988(\mathrm{~m}), 829(\mathrm{~m}), 734(\mathrm{~m}), 707(\mathrm{~s}), 619 \mathrm{~cm}^{-1}(\mathrm{~m})$; accurate mass measurement: $\mathrm{m} / \mathrm{z}$ calcd. for $\mathrm{C}_{16} \mathrm{H}_{19} \mathrm{NO}_{5}-\mathrm{H}^{-}$304.1190; found: 304.1188; elemental analysis calcd. (\%) for $\mathrm{C}_{16} \mathrm{H}_{19} \mathrm{NO}_{5} 0.25 \mathrm{H}_{2} \mathrm{O}: \mathrm{C}$ 62.03, H 6.34, N 4.52; found: C 62.07, H 6.27, N 4.60.

Methyl 1,3-dioxo-5,6-dihydro-1H,3H,4H-3a,7,8-(epiprop[2]ene[1,1,3]triyl)-5,8a-methanocyclohepta[c]furan$7(8 \mathrm{H})$-carboxylate (31). Solid $\mathrm{NaNO}_{2}(305 \mathrm{mg}, 4.4 \mathrm{mmol})$ was added portionwise in $2 \mathrm{~h}$ to a cold $\left(0{ }^{\circ} \mathrm{C}\right.$, ice-water bath) solution of amide acid $30(67 \mathrm{mg}, 0.22 \mathrm{mmol})$ in $\mathrm{Ac}_{2} \mathrm{O}(2.75 \mathrm{~mL})$ and $\mathrm{AcOH}(1.4 \mathrm{~mL})$ and the mixture was stirred at room temperature for $16 \mathrm{~h}$. The mixture was cooled to $0{ }^{\circ} \mathrm{C}$ (ice-water bath), water $(5 \mathrm{~mL})$ was added and the solution was extracted with $\mathrm{CH}_{2} \mathrm{Cl}_{2}(3 \times 8 \mathrm{~mL})$. The combined organic extracts were washed with $10 \%$ aqueous solution of $\mathrm{Na}_{2} \mathrm{CO}_{3}(3 \times 8 \mathrm{~mL})$ and water $(8 \mathrm{~mL})$, dried (anhydrous $\mathrm{Na}_{2} \mathrm{SO}_{4}$ ) and concentrated in vacuo to give anhydride $31(63.8 \mathrm{mg}$ ) as white solid. Sublimation of the above product $\left(120^{\circ} \mathrm{C} / 0.2-0.5\right.$ torr) gave the analytical sample of $31(41.5 \mathrm{mg}, 70 \%$ yield) as white solid. m.p. $184-185^{\circ} \mathrm{C}(\mathrm{EtOAc}) ;{ }^{1} \mathrm{H}-\mathrm{NMR}\left(\mathrm{CDCl}_{3}, 400 \mathrm{MHz}\right): \delta=1.92-1.93\left(\mathrm{dm},{ }^{3} \mathrm{~J}_{(\mathrm{H}, \mathrm{H})}\right.$ $\left.=2.4 \mathrm{~Hz}, 2 \mathrm{H}, 6-\mathrm{H}_{2}\right), 2.11-2.15\left[\mathrm{dm},{ }^{2} J_{(\mathrm{H}, \mathrm{H})}=12.0 \mathrm{~Hz}, 2 \mathrm{H}\right]$ and $2.18-2.22\left[\mathrm{dm},{ }^{2} J_{(\mathrm{H}, \mathrm{H})}=12.0 \mathrm{~Hz}, 2 \mathrm{H}\right]$ [4(12)-Hsyn and 4(12)-Hanti], 2.71-2.75 [m, 1H, 5-H], $3.34\left[\mathrm{t},{ }^{3} J_{(\mathrm{H}, \mathrm{H})}={ }^{4} J_{(\mathrm{H}, \mathrm{H})}=2.0 \mathrm{~Hz}, 2 \mathrm{H}, 8(11)-\mathrm{H}\right]$, $3.62\left[\mathrm{~s}, 3 \mathrm{H}, \mathrm{OCH}_{3}\right], 6.38 \mathrm{ppm}\left[\mathrm{t},{ }^{3} J_{(\mathrm{H}, \mathrm{H})}={ }^{4} J_{(\mathrm{H}, \mathrm{H})}=2.0 \mathrm{~Hz}, 2 \mathrm{H}, 9(10)-\mathrm{H}\right] ;{ }^{13} \mathrm{C}-\mathrm{NMR}\left(\mathrm{CDCl}_{3}, 100.6 \mathrm{MHz}\right)$ : $\delta=35.0\left(\mathrm{CH}_{2}, \mathrm{C} 6\right), 38.4(\mathrm{CH}, \mathrm{C} 5), 39.3\left[\mathrm{CH}_{2}, \mathrm{C} 4(12)\right], 52.2\left(\mathrm{CH}_{3}, \mathrm{OCH}_{3}\right), 56.3[\mathrm{CH}, \mathrm{C} 8(11)], 61.3[\mathrm{C}$, $\mathrm{C} 3 \mathrm{a}(8 \mathrm{a})], 72.7$ (C, C7), 136.4 [CH, C9(10)], 171.4 [C, C1(3)], 172.5 ppm (C, $\left.\mathrm{COOCH}_{3}\right)$; IR (ATR): $\check{v}=2950$ (w), $2918(\mathrm{w}), 1834(\mathrm{w}), 1770(\mathrm{~s}), 1731$ (s), $1696(\mathrm{w}), 1284(\mathrm{~m}), 1237$ (s), $1174(\mathrm{~m}), 941(\mathrm{~m}), 921$ (vs), 899 (s), $750(\mathrm{~m}), 741(\mathrm{~m}), 717 \mathrm{~cm}^{-1}(\mathrm{~s})$; accurate mass measurement: $\mathrm{m} / z$ calcd. for $\mathrm{C}_{15} \mathrm{H}_{14} \mathrm{O}_{5}+\mathrm{H}^{+}$275.0914; found: 275.0920; elemental analysis calcd. (\%) for $\mathrm{C}_{15} \mathrm{H}_{14} \mathrm{O}_{5}$ : C 65.69, $\mathrm{H}$ 5.14. Found: $\mathrm{C}$ 65.62, $\mathrm{H}$ 5.12.

7a-(Methoxycarbonyl)-3a,4,5,6,7,7a-hexahydro-1H-1,4,6-(epiethane[1,1,2] triyl)indene-4,9-dicarboxylic acid (32). A mixture of anhydride (31) $(17 \mathrm{mg}, 62 \mu \mathrm{mol})$ in water $(2 \mathrm{~mL})$ was heated under reflux for $16 \mathrm{~h}$. The solution was allowed to cool to room temperature and was concentrated in vacuo to give diacid 32 (18 mg, 99\% yield) as white solid. m.p. $174-177^{\circ} \mathrm{C}$ (water); ${ }^{1} \mathrm{H}-\mathrm{NMR}\left(\mathrm{CD}_{3} \mathrm{OD}\right): \delta=1.80\left(\mathrm{~d},{ }^{3} J_{(\mathrm{H}, \mathrm{H})}\right.$ 
$\left.=2.8 \mathrm{~Hz}, 2 \mathrm{H}, 7-\mathrm{H}_{2}\right), 1.92\left[\mathrm{~d},{ }^{2} J_{(\mathrm{H}, \mathrm{H})}=11.2 \mathrm{~Hz}, 2 \mathrm{H}, 5(8)-\mathrm{H}_{\mathrm{anti}}\right], 2.35\left[\mathrm{dd},{ }^{2} J_{(\mathrm{H}, \mathrm{H})}=11.6 \mathrm{~Hz},{ }^{3} J_{(\mathrm{H}, \mathrm{H})}=\right.$ $\left.4.0 \mathrm{~Hz}, 2 \mathrm{H}, 5(8)-\mathrm{H}_{\text {syn }}\right], 2.44-2.46(\mathrm{~m}, 1 \mathrm{H}, 6-\mathrm{H}), 2.96\left[\mathrm{t},{ }^{3} J_{(\mathrm{H}, \mathrm{H})}={ }^{4} J_{(\mathrm{H}, \mathrm{H})}=2.0 \mathrm{~Hz}, 2 \mathrm{H}, 1(3 \mathrm{a})-\mathrm{H}\right], 3.57[\mathrm{~s}$, $\left.3 \mathrm{H}, \mathrm{OCH}_{3}\right], 4.85\left(\mathrm{~s}, 12 \mathrm{H}, 2 \mathrm{COOH}\right.$ and $\left.\mathrm{CD}_{3} \mathrm{OH}\right), 6.24 \mathrm{ppm}\left[\mathrm{d}^{3} J_{(\mathrm{H}, \mathrm{H})}={ }^{4} J_{(\mathrm{H}, \mathrm{H})}=2.0 \mathrm{~Hz}, 2 \mathrm{H}, 2(3)-\mathrm{H}\right]$; ${ }^{13} \mathrm{C}-\mathrm{NMR}\left(\mathrm{CD}_{3} \mathrm{OD}\right): \delta=35.6(\mathrm{CH}, \mathrm{C} 6), 36.2\left(\mathrm{CH}_{2}, \mathrm{C} 7\right), 44.1\left[\mathrm{CH}_{2}, \mathrm{C} 5(8)\right], 52.2\left(\mathrm{CH}_{3}, \mathrm{OCH}_{3}\right), 59.3[\mathrm{CH}$, C1(3a)], 63.8 [C, C4(9)], 68.8 (C, C7a), 137.4 [CH, C2(3)], 176.6 (C, COOH), 177.2 ppm (C, $\left.\mathrm{COOCH}_{3}\right)$; IR (ATR): $\check{v}=3200-2800$ [broad band, max. at $2977(\mathrm{w})$ ], $1725(\mathrm{~m}), 1688(\mathrm{~s}), 1407(\mathrm{~m}), 1303(\mathrm{~m}), 1272(\mathrm{~m})$, $1253(\mathrm{~s}), 1243(\mathrm{~s}), 1190(\mathrm{~m}), 1102(\mathrm{~m}), 1017(\mathrm{~m}), 935 \mathrm{~cm}^{-1}(\mathrm{~m})$; accurate mass measurement: $\mathrm{m} / z$ calcd. for $\mathrm{C}_{15} \mathrm{H}_{16} \mathrm{O}_{6}-\mathrm{H}^{-}$291.0874; found: 291.0878; elemental analysis calcd. (\%) for $\mathrm{C}_{15} \mathrm{H}_{16} \mathrm{O}_{6}$ : $\mathrm{C} 61.64$, H 5.52 .

$X$-ray Crystal-structure determination of compound 24. A colorless prism-like specimen of $\mathrm{C}_{32} \mathrm{H}_{33} \mathrm{NO}_{7}$, approximate dimensions $0.050 \mathrm{~mm} \times 0.080 \mathrm{~mm} \times 0.467 \mathrm{~mm}$, was used for the X-ray crystallographic analysis. The X-ray intensity data were measured on a D8 Venture system equipped with a multilayer monochromator and a Mo microfocus $(\lambda=0.71073 \AA)$. The frames were integrated with the Bruker SAINT software package $[14,15]$ using a narrow-frame algorithm. The integration of the data using an orthorhombic unit cell yielded a total of 20021 reflections to a maximum $\theta$ angle of $26.41^{\circ}(0.80 \AA$ resolution), of which 5444 were independent (average redundancy 3.678, completeness $=99.7 \%$, $\left.\mathrm{R}_{\text {int }}=11.85 \%, \mathrm{R}_{\text {sig }}=12.14 \%\right)$ and $3454(63.45 \%)$ were greater than $2 \sigma\left(\mathrm{F}^{2}\right)$. The final cell constants of $a=8.2651$ (3) $\AA, b=11.0782(6) \AA, c=29.0497(15) \AA$, volume $=2659.9(2) \AA^{3}$, are based upon the refinement of the XYZ-centroids of reflections above $20 \sigma(\mathrm{I})$. Data were corrected for absorption effects using the multi-scan method (SADABS) [16]. The calculated minimum and maximum transmission coefficients (based on crystal size) are 0.6511 and 0.7454 . The structure was solved and refined using the Bruker SHELXTL software package [17], using the space group P 2121 21, with Z = 4 for the formula unit, $\mathrm{C}_{32} \mathrm{H}_{33} \mathrm{NO}_{7}$. The final anisotropic full-matrix least-squares refinement on $\mathrm{F}^{2}$ with 364 variables converged at $R_{1}=5.60 \%$, for the observed data and $w R_{2}=10.62 \%$ for all data. The goodness-of-fit was 1.016. The largest peak in the final difference electron density synthesis was $0.270 \mathrm{e}^{-3}$ and the largest hole was -0.244 e $\AA^{-3}$ with an RMS deviation of $0.060 \mathrm{e}^{-3}$. On the basis of the final model, the calculated density was $1.357 \mathrm{~g} \mathrm{~cm}^{-3}$ and $\mathrm{F}(000), 1152 \mathrm{e}$.

\section{Conclusions}

An alternative synthesis of polycycle $\mathbf{1 3}$ has been developed by using methoxymethyl, instead of benzyloxymethyl, as a hydroxyl-protecting group. The overall yield of both synthetic sequences is of the same order. The advantage of the new synthetic sequence derives from the fact that alcohols 19 and 20 could be separated by silica gel column chromatography and the rest of the synthesis could be carried out with the main syn-stereoisomer 19. One of the diastereomers (24) formed in the reaction of iodide $\mathbf{2 3}$ with methyl 2-oxocyclopentanecarboxylate could be isolated and its structure established by X-ray diffraction analysis. Additionally, the selective hydrolysis of the imide function of 13 to the corresponding diacid has been achieved through a non-standard procedure, whose key-step consists of the conversion of amide acid 30 to anhydride 31 on reaction with $\mathrm{NaNO}_{2}$ in a 1:1 mixture of $\mathrm{AcOH} / \mathrm{Ac}_{2} \mathrm{O}$. We hope these products may serve as new scaffolds for the synthesis of potentially active compounds.

Supplementary Materials: Supplementary materials are available online. IR and NMR spectra of all new compounds.

Acknowledgments: We thank the Ministerio de Economía y Competitividad (MINECO: SAF2014-57094-R), FEDER and the Generalitat de Catalunya (2014SGR1189) for financial support, CCiTUB for NMR and MS facilities, and the Institut de Química Avançada de Catalunya for elemental analyses.

Author Contributions: T.G. and A.O. have performed the experimental work. M.F.-B. carried out the X-ray diffraction study, and P.C. proposed and supervised the work and wrote the paper.

Conflicts of Interest: The authors declare no conflict of interest. 


\section{References}

1. Camps, P.; Gómez, T.; Monasterolo, C. An Entry to Functionalized 2,8-Ethanonoradamantane Derivatives. J. Org. Chem. 2012, 77, 11270-11282. [CrossRef] [PubMed]

2. Camps, P.; Gómez, T.; Otermin, A. Improved synthesis of 5-(t-butyldimethylsilyloxymethyl)-2-methyl-5,6dihydrocyclopenta[c]pyrrole-1,3(2H,4H)-dione from $N$-methylmaleimide. Arkivoc 2014, ii, 103-119.

3. Camps, P.; Gómez, T.; Otermin, A. Improved synthesis of a functionalized 2,8-Ethanonoradamantane derivative. Tetrahedron 2014, 70, 5190-5196. [CrossRef]

4. Bunnage, M.E.; Nicolaou, K.C. Enediyne generation by a retro-Diels-Alder reaction. Angew. Int. Ed. Engl. 1996, 35, 1110-1112. [CrossRef]

5. Bunnage, M.E.; Nicolaou, K.C. The oxide anion accelerated retro-Diels-Alder reaction. Chem. Eur. J. 1997, 3, 187-191. [CrossRef] [PubMed]

6. CCDC 1547636 contains the supplementary crystallographic data for compound 24 . These data can be obtained free of charge via http:/ / www.ccdc.cam.ac.uk/conts/retrieving.html (or from the CCDC, 12 Union Road, Cambridge CB2 1EZ, UK; Fax: +44-1223-336033; E-mail: deposit@ccdc.cam.ac.uk).

7. Angeles, A.R.; Waters, S.P.; Danishefsky, S.J. Total Syntheses of (+)- and (-)-Peribysin E. J. Am. Chem. Soc. 2008, 130, 13765-13770. [CrossRef] [PubMed]

8. Luche, J.L.; Gemal, A.L. Lanthanoids in organic synthesis. 5. Selective reductions of ketones in the presence of aldehydes. J. Am. Chem. Soc. 1979, 101, 5848-5849. [CrossRef]

9. Knapp, S.; Ornaf, R.M.; Rodriques, K.E. Synthesis of conduritol a from benzoquinone using 9-benzyloxymethoxyanthracene as a protecting and directing group. J. Am. Chem. Soc. 1983, 105, 5494-5495. [CrossRef]

10. Shang, M.; Warrener, R.N.; Butler, D.N.; Murata, Y.; Margetić, D. Synthesis of bis-peptides attached on poly $[n]$ norbornene molecular scaffolds with well-defined relative positions and distances. Mol. Divers. 2011, 15, 541-560. [CrossRef] [PubMed]

11. White, E.H. N-Alkyl-N-nitrosoamides. II. A new method for the deamination of aliphatic amines. J. Am. Chem. Soc. 1955, 77, 6011-6014. [CrossRef]

12. Samor, C.; Guerrini, A.; Varchi, G.; Baretta, G.L.; Fontana, G.; Bombardelli, E.; Carenini, N.; Zunino, F.; Bertucci, C.; Fiori, J.; et al. The Role of Polyamine Architecture on the Pharmacological Activity of Open Lactone Camptothecin-Polyamine Conjugates. Bioconj. Chem. 2008, 19, 2270-2279. [CrossRef] [PubMed]

13. Areces, P.; Pozo, M.C.; Roman, E.; Serrano, J.A. Synthesis of polycyclic systems via Diels-Alder reactions of sugar-derived dienes. Heterocycles 2000, 53, 81-92. [CrossRef]

14. APEX3, version 2016-1-0; Bruker AXS Inc.: Madison, WI, USA, 2016.

15. SAINT, version 8.35A; Bruker AXS Inc.: Madison, WI, USA, 2016.

16. SADABS, version 2014/5; Bruker AXS Inc.: Madison, WI, USA, 2016.

17. Sheldrick, G.M. Crystal structure refinement with SHELXL. Acta Crystallogr. Sect. C 2015, 71, 3-8. [CrossRef] [PubMed]

Sample Availability: Samples are not available. 